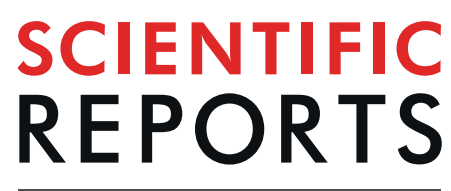

\title{
Life-span extension by pigmented rice bran in the model yeast Saccharomyces cerevisiae
}

\author{
Pitchapat Sunthonkun ${ }^{1}$, Rinsai Palajai ${ }^{1}$, Pichayada Somboon ${ }^{1}$, Chua Lee Suan $\mathbb{D}^{2}$, \\ Malyn Ungsurangsri ${ }^{3}$ \& Nitnipa Soontorngun ${ }^{1 *}$
}

Benefits of whole grains as dietary supplements and active ingredients in health products have been promoted. Despite being neglected as an agricultural byproduct of polished rice, pigmented rice bran has emerged as a promising source of natural anti-aging compounds. Indeed, the extract of red rice bran Hom Dang cultivar contained rich phenolic acids and flavonoids. It displayed high antioxidant activities in vitro and in vivo assays. Using yeast model, extract and bioactive compounds, quercetin and protocatechuic acid found in the rice bran pericarp, effectively reduced levels of intracellular reactive oxygen species (ROS), restored plasma membrane damages and prolonged life-span of pre-treated wild-yeast cells. Importantly, these molecules modulated life span-extension through a mechanism of ROS reduction that resembles to that operated under the highly conserved Tor1- and Sir2-dependent signaling pathways, with the human homologs TORC1 and SIRT1, respectively. The key longevity factors Sch9 and Rim15 kinases, Msn2/4 regulators and a novel transcription factor Asg1, the antioxidant enzymes superoxide dismutases and glutathione peroxidases played important role in mediating longevity. Yeast clearly provides an instrumental platform for rapid screening of compounds with anti-aging efficacies and advances knowledge in the molecular study of ageing.

Due to its genetic simplicity, rapid growth, ease of cultivation, the yeast Saccharomyces cerevisiae has many useful applications including in winemaking, baking, and brewing ${ }^{1,2}$. S. cerevisiae has a well-characterized genome with conserved genetic pathways that resemble those of mammalian eukaryotic cells, which makes it a eukaryotic model of choice for gaining insights into the molecular and cellular biology of higher organisms, including humans. Particularly, it is a useful model to study cellular responses to oxidants owing to several conserved antioxidant enzymes. S. cerevisiae produces endogenous reactive oxygen species (ROS) via physiological respiration of electron transport chain activity in mitochondria ${ }^{3}$. Exogenous sources of ROS include UV light, air pollution, inflammation and ionizing radiation ${ }^{4,5}$. ROS/RNS consist of free radicals such as superoxide $\left(\mathrm{O}_{2}{ }^{--}\right)$, peroxynitrite $\left(\mathrm{ONOO}^{\bullet-}\right)$, hydroxyl $\left(\mathrm{OH}^{\bullet}\right)$, and non-radicals such as hydrogen peroxide $\left(\mathrm{H}_{2} \mathrm{O}_{2}\right)^{6}$. Oxidative stress results from an imbalance between oxidants, such as ROS, and antioxidants in favor of the oxidants, leading to macromolecular damages of proteins, lipids, and/or $\mathrm{DNA}^{7}$, which could trigger apoptotic cell death ${ }^{8}$. Several reports have revealed that accumulation of ROS leads to the development of human diseases such as cancer, brain (Parkinson, Alzheimer's and migraine), cardiovascular, and inflammatory diseases ${ }^{4}$.

Antioxidant molecules are commonly present in the form of natural plant extracts ${ }^{9}$. Rice (Oryza sativa $\mathrm{L}$.) is one of the most important grain foods consumed worldwide and contains a range of bioactive compounds with antioxidant potential ${ }^{10,11}$.The amount of antioxidants found in rice, however, differs depending on grain fractions, e.g. whole grain, bran, husk, germ and endosperm, grain colors, grain types, e.g. Catahoula, Cheniere, Caffey, and the extraction processes ${ }^{12}$. Most phytochemicals, such as tocopherols, tocotrienols (vitamin E) and $\gamma$-oryzanol, in cereals are present in the bran (containing the pericarp tissue and aleurone layer) and germ fractions, and are available in lipophilic, hydrophobic and insoluble forms with potential health benefits ${ }^{13,14}$. The USDA National Small Grains Collection (NSGC) has classified rice bran by color into seven classes: white, light-brown, speckled brown, brown, red, variable purple, and purple. Anthocyanins, proanthocyanins and many other phenolic

${ }^{1}$ Division of Biochemical Technology, School of Bioresources and Technology, King Mongkut's University of Technology Thonburi, Bangkok, 10150, Thailand. ${ }^{2}$ Metabolites Profiling Laboratory, Institute of Bioproduct Development, Universiti Teknologi Malaysia, 81310 UTM Skudai, Johor Bahru, Johor, Malaysia. ${ }^{3}$ Research and Development division, S\&J International Enterprises Public Company Limited, Bangkok, Thailand. *email: nitnipa. soo@kmutt.ac.th 


\begin{tabular}{|c|c|c|c|c|}
\hline \multirow{2}{*}{\begin{tabular}{|l|} 
Rice bran \\
Extraction
\end{tabular}} & \multirow{2}{*}{$\begin{array}{l}\text { a'TPC } \\
\text { (mg GAE/g } \\
\text { DW) }\end{array}$} & \multicolumn{2}{|c|}{${ }^{b} E C_{50}(\mathrm{mg} / \mathrm{mL})$} & \multirow{2}{*}{\begin{tabular}{|l|} 
eFRAP \\
( $\mu$ moleFe(II)/g extract)
\end{tabular}} \\
\hline & & ${ }^{\mathrm{c}} \mathrm{DPPH}$ & dABTS & \\
\hline \multicolumn{5}{|c|}{ Propylene Glycol } \\
\hline Hom Dang & $1.44 \pm 0.08$ & $0.47 \pm 0.25$ & $0.006 \pm 0.001$ & $5.85 \pm 0.20 \times 10^{6}$ \\
\hline Kum Doi Saket & $1.09 \pm 0.06$ & $0.59 \pm 0.09$ & $0.008 \pm 0.002$ & $7.29 \pm 0.50 \times 10^{6 *}$ \\
\hline \multicolumn{5}{|l|}{ Ethanolic } \\
\hline Hom Dang & $1.69 \pm 0.12$ & $0.35 \pm 0.20$ & $0.007 \pm 0.002 *$ & $6.85 \pm 1.30 \times 10^{6}$ \\
\hline Kum Doi Saket & $1.20 \pm 0.16$ & $0.45 \pm 0.50$ & $0.013 \pm 0.012$ & $6.39 \pm 0.60 \times 10^{6}$ \\
\hline Ascorbic acid & & $0.02 \pm 0.13^{*}$ & N.I & N.I \\
\hline
\end{tabular}

Table 1. In vitro antioxidant properties of Thai pigmented rice bran extracts. ${ }^{\text {a }}$ Total phenolic content in milligram of gallic acid in $1 \mathrm{~g}$ of pigmented rice bran extract. ${ }^{\mathrm{b}} \mathrm{EC}_{50}=$ the concentration of a compound that

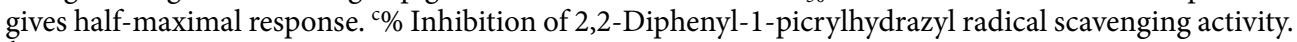
${ }^{\mathrm{d}} 50 \%$ 2,2'-azino-bis-3-ethylbenzthiazoline-6-sulphonic acid scavenging activity. ${ }^{\mathrm{e}}$ Ferric reducing/antioxidant power in milligram of Trolox equivalent in $1 \mathrm{~g}$ of pigmented rice bran extract. ${ }^{\mathrm{f}} \mathrm{N} . \mathrm{I}=$ Not identified. Values are presented as means \pm S.D. Statistical significance was considered at: $* p<0.01$.

compounds have been reported in red and black rice varieties ${ }^{15}$, in addition to flavonoids including flavonols, flavan-3-ols, flavones, and flavanones ${ }^{16}$. Phenolic compounds such as ferulic acid, $p$-coumaric acid, vanillic acid, caffeic acid, syringic acid, and sinapinic acid have also been found in some varieties of black rice ${ }^{17}$. The most abundant phenolic acids in rice bran of black and red rice are ferulic acid and $p$-coumaric acid, which accounts for almost $50-65 \%$ of total phenolic acids ${ }^{18,19}$. In addition, the flavonoids include flavonols, flavan-3-ols, flavones and flavanones are reported ${ }^{16}$. High amount of phytochemicals with antioxidant capacity have a wide range of therapeutic effects against chronic diseases ${ }^{20,21}$.

In S. cerevisiae, biological aging is evolutionarily conserved and controlled by a network of proteins kinases in many signaling pathways. The network of chronological aging integrates the pro-aging TORC1 (target of rapamycin complex pathway), which stimulates the pro-aging protein kinase Sch9, and pro-aging SIR. In contrast, it includes antagonist mechanisms including the anti-aging Snf1 (sucrose non-fermenting) pathway, the anti-aging ATG (autophagy) pathway, and the anti-aging protein kinase Rim15, inhibited by the TORC1, PKA, and PKH1/2 pathways. The protein kinase Rim 15 phosphorylates Msn2/4 transcription factors activate gene expression by binding to the stress response element (STRE) to control environmental stresses, including heat shock, oxidative stress, and nutrient starvation. Msn2/4 transcription factors modulate manganese superoxide dismutase encoding-SOD2 genes in defense against toxic ROSs ${ }^{22}$. Two SODs, namely $\mathrm{Cu}-\mathrm{Zn}$ dependent cytosolic and intermembrane space Sod 1 and Mn-dependent Sod2, which are localized it the mitochondrial matrix guard against mitochondrial superoxide production ${ }^{20-23}$. In addition, $S$. cerevisiae has glutathione peroxidases to protect cells from oxidative damage ${ }^{23}$. GPX1 gene encodes an enzyme, Gpx1, that functions in the detoxification of hydrogen peroxide particularly by catalyzing the reduction of hydrogen peroxide to water ${ }^{23}$. GPX2 gene encodes an atypical 2-Cys peroxiredoxin or phospholipid hydroperoxide glutathione peroxidase that applies thioredoxin as an electron donor ${ }^{24}$. In aerobic metabolism, antioxidant functions of the reduced glutathione GSH can be metabolized by glutathione peroxidases $(\mathrm{Gpx})$ in the cytosol and mitochondria ${ }^{23,25}$.

Despite extensive studies on the existence of antioxidants in pigmented rice brans, little is known regarding the role they play in longevity. The aims of this study were to apply the yeast $S$. cerevisiae gene knockout system to identify new longevity factors and to determine the antioxidant and anti-aging potential of pigmented rice bran extract and compounds with the focus on their beneficial effect on chronological lifespan. Some candidates for anti-aging drugs, such as spermidine and resveratrol, and anti-aging interventions, such as caloric restriction, have been identified and explored in yeast ${ }^{26}$. Insights into the involved mechanism will help us to further explore other potential anti-aging agents.

\section{Results}

Total phenolic content (TPC) and antioxidant activities of pigmented rice bran extract. The antioxidant activities of bran extracts from two pigmented rice varieties (red and black) were determined by in vitro assays. These included the inhibition of 2, 2-diphenyl-1-picrylhydrazyl (DPPH) using 2,2'-azino-bis-3 ethylbenzthiazoline-6-sulphonic acids (ABTS) as an oxidant and FRAP (Ferric reducing antioxidant power). Total phenolic contents were also determined. The extraction was performed using two polar solvents, ethanol and propylene glycol, separately. The TPC of ethanolic rice bran extracts of Hom Dang (red rice) and Kum Doi Saket (black rice) was in the range of $1.20 \pm 0.16$ to $1.69 \pm 0.12 \mathrm{mg} \mathrm{GAE} / \mathrm{g}$ extract, while propylene glycolic extracts ranged from $1.09 \pm 0.06$ to $1.44 \pm 0.08 \mathrm{mg} \mathrm{GAE} / \mathrm{g}$ extract (Table 1). Of both rice varieties, the highest TPC was obtained from Hom Dang rice bran ethanolic extract (Table 1). Its DPPH free radical scavenging activity also showed the lowest $\mathrm{EC}_{50}(0.35 \pm 0.20 \mathrm{mg} / \mathrm{mL})$. The ABTS scavenging assays also showed similar antioxidant capacity for all rice bran extracts tested (Table 1). Also, the propylene glycolic extract of Kum Doi Saket had the highest ferrous-chelating capacity (FRAP) of 7.29 $\pm 0.50 \times 10^{6} \mu$ mole Fe(II)/g extract followed by the ethanolic Hom Dang rice bran and Kum Doi Saket rice bran extracts (Table 1). High total anthocyanin content involved in high ferrous-chelating capacity (FRAP), in the black rice bran extract is proposed to be responsible for the antioxidant activity among other pigmented rice bran extract. Overall, Hom Dang rice bran extract showed the best TPC and scavenging activity (Table 1). 
Determination of antioxidant compounds of pigmented rice bran extract. The UV spectra of the hydroxybenzoic acids were relevant and used to elucidate their chemical structures (Table 2). Single absorption peaks appeared in the UV spectra of compounds such as $p$-coumaric acid providing a $[\mathrm{M}-\mathrm{H}]^{-}$anion at $\mathrm{m} / \mathrm{z} 119$ and protocatchuic acid providing a $[\mathrm{M}-\mathrm{H}]^{-}$anion at $\mathrm{m} / \mathrm{z} 109$, which have symmetrical chemical structures. However, the position and number of hydroxyl groups on the aromatic rings also had a significant effect on wavelength shift. The ferulic acid provided $[\mathrm{M}-\mathrm{H}]^{-}$anions at $\mathrm{m} / \mathrm{z} 134, \mathrm{~m} / \mathrm{z} 149, \mathrm{~m} / \mathrm{z} 178$, all of which had non-symmetrical chemical structures, a major absorption peak and a shoulder absorption under our conditions, which was inconsistent with the previous report ${ }^{16,24}$. (+)-Catechin $\left([\mathrm{M}-\mathrm{H}]^{-}\right)$yielded fragment ions at $\mathrm{m} / \mathrm{z} 205$ and $\mathrm{m} / \mathrm{z} 245$, which were produced by the loss of a $(\mathrm{CH})_{2} \mathrm{OH}$ group. Also, $(-)$ ESI-MS/MS spectra of the procyanidin dimer and procyanidintrimer $\left([\mathrm{M}-\mathrm{H}]^{-}\right.$gave fragment ions at $\mathrm{m} / \mathrm{z} 695$ from rearrangement of the heterocyclic ring ${ }^{27}$, at $\mathrm{m} / \mathrm{z} 425[\mathrm{M}-\mathrm{H}]^{-}$from RDA-F of the heterocyclic ring and loss of $\mathrm{H}_{2} \mathrm{O}$, at m/z $289[\mathrm{M}-\mathrm{H}]^{-}$from cleavages between $\mathrm{C}_{4}-\mathrm{C}_{5}$ and $\mathrm{O}-\mathrm{C}_{2}$ of one pyran ring, and at $\mathrm{m} / \mathrm{z} 289\left([\mathrm{M}-\mathrm{H}-289]^{-}\right)$from cleavage of the interflavanic bond. Kaempferol had a fragment ion at $\mathrm{m} / \mathrm{z} 257, \mathrm{~m} / \mathrm{z} 151, \mathrm{~m} / \mathrm{z} 169$, also from the cleavage of the heterocyclic C-ring by RDA, but the fragmentation mechanism remains unclear at present. For flavonol-O-glycosides such as rutin fragment ions at $\mathrm{m} / \mathrm{z} 301, \mathrm{~m} / \mathrm{z} 179, \mathrm{~m} / \mathrm{z} 151$, and quercetin at $\mathrm{m} / \mathrm{z} 151, \mathrm{~m} / \mathrm{z} 179$, their spectra showed a deprotonated $[\mathrm{M}-\mathrm{H}]^{-}$molecule of the glycoside and an $[\mathrm{A}-\mathrm{H}]^{-}$ion corresponding to the deprotonated aglycone. The latter ion is formed by losing the rutinose moiety from the corresponding glycosides.

In summary, the ESI-MS/MS spectra analyzed showed that propylene glycolic Hom Dang rice bran extract contained many free phenolic acids including $p$-courmaric acid, protocatchuic acid, and ferulic acid (Table 2). It also contained tannin, including proanthocyanidin dimer and proanthocyanidin trimer, as well as $\alpha$-tocopherol and flavonoids, including catechin, rutin, kaempherol, isorhamnetin, and quercetin (Table 2). However, the propylene glycolic extract of Kum Doi Saket contained less bioactive compounds, only ferulic, isorhamnetin, protocatechuic acid, rutin, kaempherol, and p-courmaric acid (Table 2). Based on the LC/MS-MS analysis, Hom Dang rice bran extract contained, in comparison, a higher amount of bioactive compounds, some of which included phenolic acids and flavonoids.

Effects of pigmented rice bran extracts on $\mathrm{H}_{2} \mathrm{O}_{2}$ scavenging. S. cerevisiae is a good and easy organism for use as an in vivo model for testing the ability of antioxidants to scavenge intracellular ROS ${ }^{28}$. It was used to investigate the ability of Hom Dang rice bran extract to scavenge intracellular ROS generated by the potent oxidant $\mathrm{H}_{2} \mathrm{O}_{2}$, based on the DCF method ${ }^{29}$. The conversion of the non-fluorescent $\mathrm{DCFH}_{2}$-DA to highly fluorescent DCF is well documented and occurs in several steps. $\mathrm{DCFH}_{2}-\mathrm{DA}$ is transported across the cell membrane and deacetylated by esterases to form the non-fluorescent $\mathrm{DCFH}_{2}$. This compound is trapped inside the cells. Next, $\mathrm{DCFH}_{2}$ is converted to DCF through the action of intracellular oxidants; $\mathrm{H}_{2} \mathrm{O}_{2}, \mathrm{HO}^{\bullet}, \mathrm{ROO}^{\bullet}{ }^{30}$. Different yeast deletion strains lacking key genes in aging and antioxidant systems, namely SOD1, SOD2, GPX1,GPX2, TOR1, SIR2, RIM15, MSN2/4, ASG1, and SCH9, were pre-treated with Hom Dang rice bran extracts prior to the exposure to $5 \mathrm{mM} \mathrm{H}_{2} \mathrm{O}_{2}$.

The DCFH-DA assay showed that the ROS levels were increased after exposure to $5 \mathrm{mM} \mathrm{H}_{2} \mathrm{O}_{2}$ for all strains, especially the $\Delta$ sod 1 strain lacking the enzyme superoxide dismutase, which showed a 2 -fold increase in the ROS level (Fig. 1a). Addition of the antioxidant agents, thiourea (TU) or ascorbic acid (AA or vitamin c), did not alter the levels of ROS but contributed to the lowering of the ROS level. As shown, the ROS levels dropped to the same or lower level than under untreated $\left(\mathrm{H}_{2} \mathrm{O}_{2}\right)$ condition for all tested strains (Fig. 1a). The ROS levels in the WT, the $\Delta \operatorname{sod} 1$ and the $\Delta \operatorname{sod} 2$ strains were significantly decreased to the background ROS level after treatment with $0.01 \mathrm{mg} / \mathrm{mL}$ of the Hom Dang rice bran extract (Fig. 1a). Similarly, ROS levels in the WT, the $\Delta g p \times 1$, and the $\Delta g p \times 2$ strains were significantly decreased after treatment with $0.01 \mathrm{mg} / \mathrm{mL}$ of the Kum Doi Saket rice bran extract (Fig. 1a). Thus, the results suggested the antioxidant activity of the pigmented rice bran extracts to reduce intracellular ROS levels in mutant yeast strains lacking antioxidant enzymes.

Ability of Hom Dang rice bran extract to reduce intracellular ROS in aged yeast. Due to the high antioxidant activity of Hom Dang rice bran extract, its ability to reduce intracellular ROS was evaluated against two bioactive compounds, quercetin and protocatechuic acid, found in the rice bran (Table 2). The wild-type yeast $S$. cerevisiae strain was cultured for a total duration of 30 days. Addition of the Hom dang rice bran extract, quercetin or protocatechuic acid gradually decreased the intracellular fluorescence intensity from day 3 until day 21 , when used at a concentration of $0.01-0.1,0.008$, and $0.002 \mathrm{mg} / \mathrm{mL}$, respectively (Fig. 1b). The antioxidants could permeate through the yeast cell membrane and intercept ROS forming radicals, thereby preventing the production of different ROS compounds required to oxidize intracellular $\mathrm{DCFH}_{2}$ to the fluorescent DCF through post-diauxic and stationary phases (Fig. 1b). The efficient suppression of intracellular ROS production by the Hom dang rice bran extract, quercetin, and protocatechuic acid indicated that these agents act with strong radical scavenging potency in the intracellular environment.

Hom Dang rice bran extract treatment prevented cell membrane damage. To provide additional evidence for the aforementioned findings, we examined the membrane permeability of the pigmented rice bran extract, quercetin or protocatechuic acid-pretreated $S$. cerevisiae cells using propidium iodide dye $\left(\mathrm{Pi}^{+}\right)^{31}$. The exclusion of this hydrophilic red standard dye from cells with intact membranes is indicative if its integrity, whereas severely damaged cell membranes allow the dye $\mathrm{in}^{32}$. It is used to determine the membrane impermeability. Results showed that the reduction of $\mathrm{Pi}^{+}$dye by the Hom Dang rice bran extract is time and concentration dependent. At dayl, the untreated and pre-treated S. cerevisiae cells had low levels of fluorescence intensity, indicating minimal cell membrane damage (Fig. 1d). The fluorescence was also shown for the control, $65^{\circ} \mathrm{C}$-heated cells, at day 1 and day 7. By day 7 of culturing, $\mathrm{Pi}^{+}$was strongly fluorescent in the untreated condition as cells aged (Fig. 1d). However, the fluorescence was dramatically reduced in cells treated with $0.1 \mathrm{mg} / \mathrm{mL}$ of the Hom Dang 


\begin{tabular}{|c|c|c|c|c|}
\hline Identified Compounds & Structures & Rt (min) & {$[\mathrm{M}-\mathrm{H}]^{-}$} & \begin{tabular}{|l|} 
Rice bran \\
cultivars
\end{tabular} \\
\hline \multicolumn{5}{|l|}{ Phenolic acids } \\
\hline$p$-Courmaric acid & & 7.59 & 119 & HD,KD \\
\hline Protocatchuic acid & & 5.66 & 109 & HD,KD \\
\hline Ferulic acid & & 7.91 & $134,149,178$ & HD, KD \\
\hline \multicolumn{5}{|l|}{ Flavonoid } \\
\hline Proanthocyanidin dimer & & 6.30 & 425,289 & HD \\
\hline Proanthocyanidin trimer & & 6.41 & 695 & HD \\
\hline Catechin & & 6.41 & 245,205 & HD \\
\hline Rutin & & 7.93 & $301,179,151$ & $\mathrm{HD}, \mathrm{KD}$ \\
\hline Kaemferol & & 8.99 & $257,151,169$ & $\mathrm{HD}, \mathrm{KD}$ \\
\hline Isorhamnetin & & 10.26 & 300 & HD,KD \\
\hline Quercetin & & 9.51 & 151,179 & HD \\
\hline$\alpha$-tocopherol & & 5.43 & $431,343,205$ & HD,KD \\
\hline
\end{tabular}

Table 2. Analysis of bioactive compounds in pigmented rice bran extracts of Hom Dang rice bran (HD) and Kum Doi Saket rice bran (KD). 

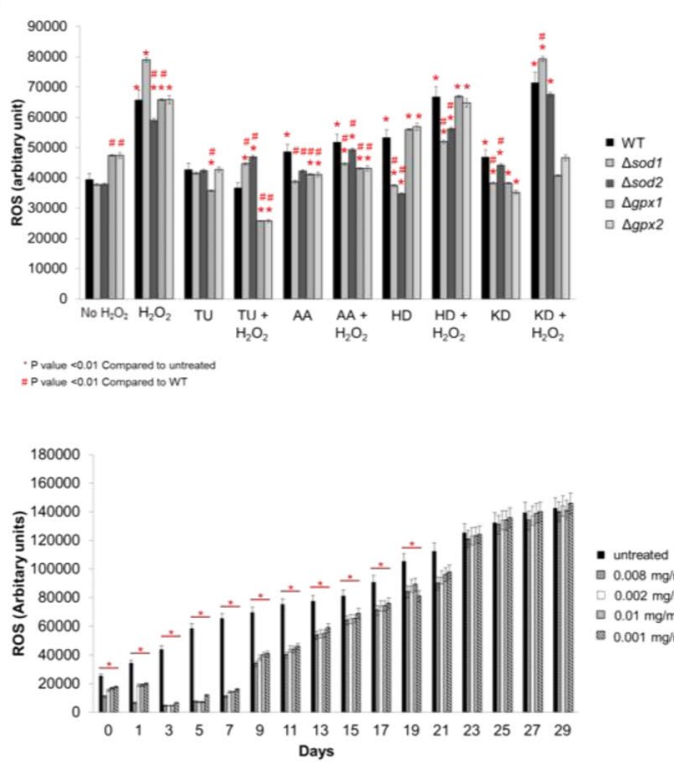

Days

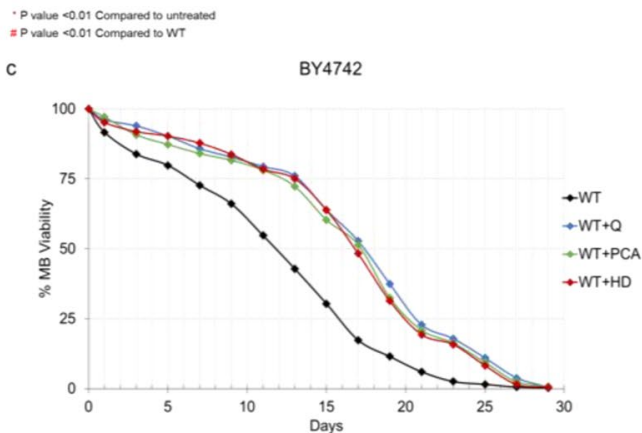

- untreated

WT+PCA

WT+HD
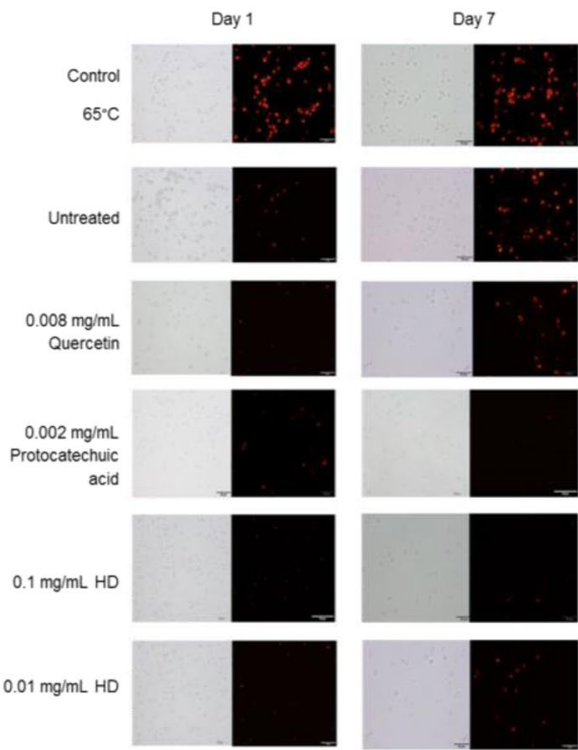

$0.002 \mathrm{mg} / \mathrm{mL}$ PCA

$0.01 \mathrm{mg} / \mathrm{mL}$ HO

$=0.001 \mathrm{mg} / \mathrm{mL}$ HD

Figure 1. (a) Quantification of cellular ROS levels via the fluorescent DCFH-DA assay in the S. cerevisiae wildtype (WT) and mutant yeast cells. The WT, the $\Delta \operatorname{sod} 1$ and the $\Delta \operatorname{sod} 2$ strains were treated with $0.01 \mathrm{mg} / \mathrm{mL}$ Hom Dang rice bran extract (HD). The WT the $\Delta g p x 1$ and the $\Delta g p x 2$ strains were treated with $0.01 \mathrm{mg} / \mathrm{mL}$ Kum Doi Saket rice bran extract (KD). After, cells were exposed to hydrogen peroxide $\left(\mathrm{H}_{2} \mathrm{O}_{2}\right)$ to induce generation of ROS. Endogenous ROS levels of cells were measured by a fluorometric assay using $2^{\prime}, 7^{\prime}$-dichlorofluorescein diacetate (DCFH-DA) as a ROS indicator. Error bars represented standard error of the mean (SEM) $\left(* p,{ }^{*} p<0.01\right.$, two-tailed Student's t test compared to untreated condition and wild-type, respectively). TU referred to thiourea and AA referred to ascorbic acid. (b) Quantification of intracellular ROS levels in the chronological ageing S. cerevisiae WT strain treated with antioxidants $0.008 \mathrm{mg} / \mathrm{mL}$ of quercetin (Q), $0.002 \mathrm{mg} / \mathrm{mL}$ of protocatechuic acid (PCA), $0.1 \mathrm{or} 0.01 \mathrm{mg} / \mathrm{mL}$ Hom Dang rice bran extract (HD) for 29 days. The ROS levels were analyzed using the fluorescent probe DCFH-DA. Error bars represented standard error of the mean (SEM) $\left({ }^{*} p<0.01\right.$, two-tailed Student's t test compared to untreated condition). (c) Survival curves of chronologically aging yeast cells from day 0 to day 30 were shown for the wild-type $S$. cerevisiae BY4742. Pre-treatment with antioxidants quercetin (Q) or protocatechuic acid (PCA) or Hom Dang rice bran extract (HD) showed increasing life-span extension only in the WT strain. ${ }^{*} p<0.01$, two-tailed Student's t test compared to untreated condition). (d) Plasma membrane permeability of the ageing S. cerevisiae WT strain at day 1 and day 7 was examined. The WT strain was either chronologically grown at $30^{\circ} \mathrm{C}$ without antioxidants (untreated negative control), or treated with antioxidants $0.008 \mathrm{mg} / \mathrm{mL}$ of quercetin $(\mathrm{Q}), 0.002 \mathrm{mg} / \mathrm{mL}$ of protocatechuic acid (PCA), 0.1 or $0.01 \mathrm{mg} / \mathrm{mL}$ Hom Dang rice bran extract (HD) or heated to $65^{\circ} \mathrm{C}$ for $10 \mathrm{~min}$. (positive control). Cells were stained with propidium iodide and, the membrane permeability was analyzed by fluorescence microscopy.

rice bran (HD) or $0.002 \mathrm{mg} / \mathrm{mL}$ of the protocatechuic acid (PCA) (Fig. 1d), which correlates well with low ROS levels of cells and is highly efficient against intracellular radicals (Fig. $1 \mathrm{a}-\mathrm{c})$. A lower concentration of $(0.01 \mathrm{mg} / \mathrm{mL})$ Hom Dang rice bran extract (HD) and $0.008 \mathrm{mg} / \mathrm{mL}$ of quercetin (Q) had less effect on membrane permeability and only protected yeast cells at day1 (Fig. 1d). Overall the results suggested that increased intracellular ROS accumulation in aged cells correlates with increased plasma membrane permeability (Fig. 1b,d). Similarly, decreased ROS accumulation in S. cerevisiae cells, when incubated with the Hom Dang rice bran extract (HD) and corresponding pure antioxidants, protocatechuic acid (PCA) and quercetin (Q), supported their mechanistic involvement in ROS scavenging and activation of stress response systems during the aging process. 


\begin{tabular}{|c|c|c|c|c|c|c|c|c|c|c|c|c|c|c|c|c|c|}
\hline \multirow[b]{2}{*}{ Strain } & \multirow[b]{2}{*}{ Day } & \multicolumn{4}{|c|}{$\begin{array}{l}\text { \% Normalized MB Viability compared } \\
\text { to day } 0\end{array}$} & \multicolumn{4}{|c|}{$\begin{array}{l}\text { \% Difference in MB Viability normalized } \\
\text { to day } 0\end{array}$} & \multicolumn{4}{|c|}{$\begin{array}{l}\text { \% Difference in MB Viability normalized to } \\
\text { untreated condition }\end{array}$} & \multicolumn{4}{|c|}{$\begin{array}{l}\text { \% Difference in MB Viability normalized } \\
\text { to WT }\end{array}$} \\
\hline & & untreated & \begin{tabular}{|l|}
$\begin{array}{l}\text { Quercetin } \\
\text { (Q) }\end{array}$ \\
\end{tabular} & PCA & \begin{tabular}{|l} 
HD \\
extract
\end{tabular} & untreated & \begin{tabular}{|l} 
Quercetin \\
(Q)
\end{tabular} & PCA & $\begin{array}{l}\text { HD } \\
\text { extract }\end{array}$ & untreated & \begin{tabular}{|l|} 
Quercetin \\
(Q)
\end{tabular} & PCA & $\begin{array}{l}\text { HD } \\
\text { extract }\end{array}$ & untreated & \begin{tabular}{|l|}
$\begin{array}{l}\text { Quercetin } \\
\text { (Q) }\end{array}$ \\
\end{tabular} & PCA & \begin{tabular}{|l} 
HD \\
extract
\end{tabular} \\
\hline \multirow{4}{*}{ WT } & 0 & 100 & 100 & 100 & 100 & 0 & 0 & 0 & 0 & 0 & 0 & 0 & \begin{tabular}{|l|l|} 
\\
\end{tabular} & 0 & \begin{tabular}{|l|l|} 
\\
\end{tabular} & \begin{tabular}{|l|l|} 
\\
\end{tabular} & 0 \\
\hline & 7 & 73 & 86 & 84 & 88 & -27 & -14 & -16 & -12 & 0 & $18^{*}$ & $16^{*}$ & $21^{*}$ & 0 & 0 & 0 & 0 \\
\hline & \begin{tabular}{|l|}
13 \\
\end{tabular} & 43 & 76 & \begin{tabular}{|l|}
72 \\
\end{tabular} & \begin{tabular}{|l|}
75 \\
\end{tabular} & -57 & -24 & \begin{tabular}{|l|}
-28 \\
\end{tabular} & -25 & 0 & \begin{tabular}{|l|l|}
$77^{*}$ \\
\end{tabular} & 69* & \begin{tabular}{|l|l}
$75^{*}$ \\
\end{tabular} & 0 & 0 & 0 & 0 \\
\hline & 17 & 17 & 53 & 51 & 48 & -83 & -47 & -49 & -52 & 0 & $205^{*}$ & $197^{*}$ & $179^{*}$ & 0 & 0 & 0 & 0 \\
\hline \multirow{4}{*}{$\Delta s i r 2$} & 0 & 100 & 100 & 100 & 100 & 0 & 0 & 0 & 0 & 0 & 0 & 0 & 0 & 0 & 0 & 0 & 0 \\
\hline & 7 & 81 & 80 & 80 & 80 & -19 & -20 & -20 & -20 & 0 & -1 & -1 & -2 & $12^{*}$ & $-7^{*}$ & -5 & -9 \\
\hline & 13 & 63 & 63 & 65 & 63 & -37 & -37 & -35 & -37 & 0 & 1 & 4 & 1 & $46^{*}$ & $-17^{*}$ & $-10^{*}$ & $-16^{*}$ \\
\hline & 17 & 29 & 28 & 26 & 28 & -71 & -72 & -74 & -72 & 0 & -4 & -7 & -3 & $65^{*}$ & $-48^{*}$ & $-49^{*}$ & $-43^{*}$ \\
\hline \multirow{4}{*}{$\Delta$ torl } & 0 & 100 & 100 & 100 & 100 & 0 & 0 & 0 & 0 & 0 & 0 & 0 & 0 & 0 & 0 & 0 & 0 \\
\hline & 7 & 79 & 79 & 81 & 78 & -21 & -21 & -19 & -22 & 0 & 0 & 1 & -2 & 9* & $-8^{*}$ & -4 & -11 \\
\hline & 13 & 60 & 57 & 62 & 61 & -40 & -43 & -38 & -40 & 0 & -6 & 2 & 0 & $41^{*}$ & $-26^{*}$ & $-15^{*}$ & $-19 *$ \\
\hline & 17 & 30 & 28 & 30 & 31 & -70 & -72 & -70 & -69 & 0 & -7 & -3 & 2 & $75^{*}$ & $-47^{*}$ & $-43^{*}$ & $-36^{*}$ \\
\hline \multirow{4}{*}{$\Delta \operatorname{sch} 9$} & 0 & 100 & 100 & 100 & 100 & 0 & 0 & 0 & 0 & 0 & 0 & 0 & 0 & 0 & 0 & 0 & 0 \\
\hline & 7 & 78 & \begin{tabular}{|l|}
79 \\
\end{tabular} & \begin{tabular}{|l|}
81 \\
\end{tabular} & \begin{tabular}{|l|l|}
79 \\
\end{tabular} & -22 & -21 & \begin{tabular}{|l|}
-19 \\
\end{tabular} & -21 & 0 & 0 & 3 & \begin{tabular}{|l|l|} 
& \\
\end{tabular} & 8* & $-8^{*}$ & -4 & -10 \\
\hline & 13 & 61 & 62 & 62 & 61 & -39 & -38 & -38 & -40 & 0 & 3 & 1 & 0 & $41^{*}$ & $-18^{*}$ & $-15^{*}$ & $-19^{*}$ \\
\hline & \begin{tabular}{|l|}
17 \\
\end{tabular} & 30 & 32 & 34 & 31 & -70 & -68 & -66 & -69 & 0 & 7 & 10 & 2 & \begin{tabular}{|l|l}
$75 *$ \\
\end{tabular} & $-39 *$ & $-35^{*}$ & $-36^{*}$ \\
\hline \multirow{4}{*}{$\Delta \operatorname{rim} 15$} & 0 & 100 & 100 & 100 & 100 & 0 & 0 & 0 & 0 & 0 & 0 & 0 & 0 & 0 & 0 & 0 & 0 \\
\hline & 7 & 62 & 74 & 75 & \begin{tabular}{|l|}
79 \\
\end{tabular} & -38 & -26 & -25 & -21 & 0 & $19^{*}$ & $20^{*}$ & $27^{*}$ & $-14^{*}$ & $-14^{*}$ & $-11^{*}$ & $-10^{*}$ \\
\hline & \begin{tabular}{|l|}
13 \\
\end{tabular} & 32 & 40 & 47 & 46 & -68 & -60 & -53 & -54 & 0 & $26^{*}$ & $46^{*}$ & 44* & $-25^{*}$ & $-47^{*}$ & $-35^{*}$ & $-39 *$ \\
\hline & \begin{tabular}{|l|}
17 \\
\end{tabular} & 15 & 19 & 22 & 22 & -85 & -81 & -78 & -78 & 0 & $33^{*}$ & $53^{*}$ & $53^{*}$ & $-16^{*}$ & $-63^{*}$ & $-57^{*}$ & $-54^{*}$ \\
\hline \multirow{4}{*}{$\Delta m s n 2$} & 0 & 100 & 100 & \begin{tabular}{|l|l|}
100 \\
\end{tabular} & 100 & 0 & 0 & 0 & 0 & 0 & 0 & 0 & 0 & 0 & 0 & 0 & 0 \\
\hline & 7 & 62 & 70 & 76 & 76 & -38 & -30 & -24 & -24 & 0 & $13^{*}$ & $22^{*}$ & $22^{*}$ & $-14^{*}$ & $-18^{*}$ & $-9^{*}$ & $-13^{*}$ \\
\hline & 13 & 30 & 42 & 45 & 44 & -70 & -58 & -55 & -56 & 0 & $41^{*}$ & $52^{*}$ & \begin{tabular}{|l|l}
$48^{*}$ \\
\end{tabular} & $-30^{*}$ & $-45^{*}$ & $-37^{*}$ & $-41^{*}$ \\
\hline & \begin{tabular}{|l|}
17 \\
\end{tabular} & 10 & 18 & 23 & 20 & -90 & -82 & -77 & -80 & 0 & $78^{*}$ & $127^{*}$ & $92^{*}$ & $-41^{*}$ & $-65^{*}$ & $-55^{*}$ & $-59 *$ \\
\hline \multirow{4}{*}{$\Delta m s n 4$} & 0 & 100 & 100 & \begin{tabular}{|l|l|}
100 \\
\end{tabular} & 100 & 0 & 0 & \begin{tabular}{|l|l|} 
\\
\end{tabular} & 0 & 0 & 0 & 0 & 0 & 0 & 0 & 0 & 0 \\
\hline & \begin{tabular}{|l|}
7 \\
\end{tabular} & 60 & 70 & 76 & \begin{tabular}{|l|}
75 \\
\end{tabular} & -40 & -30 & -24 & -25 & 0 & $16^{*}$ & $26^{*}$ & $24^{*}$ & $-17^{*}$ & $-18^{*}$ & $-9 *$ & $-14^{*}$ \\
\hline & \begin{tabular}{|l|}
13 \\
\end{tabular} & 31 & 42 & 45 & 45 & -69 & -58 & -55 & -55 & 0 & $37^{*}$ & $47^{*}$ & $47^{*}$ & $-28^{*}$ & $-45^{*}$ & $-37^{*}$ & $-40^{*}$ \\
\hline & \begin{tabular}{|l|}
17 \\
\end{tabular} & 10 & \begin{tabular}{|l|}
18 \\
\end{tabular} & 23 & 21 & -90 & -82 & \begin{tabular}{|l|}
-77 \\
\end{tabular} & -79 & 0 & \begin{tabular}{|l|l|}
$78^{*}$ \\
\end{tabular} & \begin{tabular}{|l|l|}
$127 *$ \\
\end{tabular} & 103* & $-41 *$ & $-65^{*}$ & $-55^{*}$ & $-57 *$ \\
\hline \multirow{4}{*}{ Dasg1 } & 0 & 100 & 100 & 100 & 100 & 0 & 0 & 0 & 0 & 0 & 0 & 0 & 0 & 0 & 0 & 0 & 0 \\
\hline & \begin{tabular}{|l|}
7 \\
\end{tabular} & \begin{tabular}{|l|}
62 \\
\end{tabular} & 71 & 75 & 75 & -38 & -29 & -25 & -25 & 0 & \begin{tabular}{|l|l|}
$16^{*}$ \\
\end{tabular} & $22^{*}$ & $22^{*}$ & $-15^{*}$ & $-17^{*}$ & $-10^{*}$ & $-15^{*}$ \\
\hline & 13 & 32 & 44 & 45 & 45 & -68 & -56 & -55 & -55 & 0 & $36^{*}$ & $41^{*}$ & $41^{*}$ & $-25^{*}$ & $-43^{*}$ & $-37^{*}$ & $-40^{*}$ \\
\hline & \begin{tabular}{|l|}
17 \\
\end{tabular} & 10 & 17 & 20 & 19 & -90 & -83 & -80 & -81 & 0 & \begin{tabular}{|l|l|}
$69^{*}$ \\
\end{tabular} & 98* & $88 *$ & $-41 *$ & $-67^{*}$ & $-60^{*}$ & $-60^{*}$ \\
\hline
\end{tabular}

Table 3. \% Normalized MB Viability and difference in MB Viability normalized to day 0 , the untreated condition or WT of CLS assay at day $0,7,13$, and $17\left({ }^{*} p<0.0001\right)$. Q, PCA and HD referred to quercetin, protocathechuic acid and Hom Dang, respectively. Negative values indicated the decreased values.

Antioxidants and Hom Dang rice bran extract extended lifespan of wild-type yeast cells. Aging has been associated with an increase in the accumulation of ROS and a decrease in antioxidant defenses. This oxidative damage theory of aging is supported by data showing that increased scavenging of ROS by over expression of antioxidant enzymes delays aging ${ }^{31}$. The protection against oxidative stress conferred by the antioxidants and the extract of Hom Dang rice bran extract led us to analyze their effect on yeast chronological life span. $0.1 \mathrm{mg} / \mathrm{mL}$ of Hom Dang rice bran extract increased the lifespan of $S$. cerevisiae wild-type BY4742 strain significantly compared to the untreated strain (Fig. 1c and Table 3). The life-span extension was increased by 5 days at $50 \%$ viability when compared to the untreated wild-type strain (Fig. 1c). Similarly, pre-treatment with $0.002 \mathrm{mg} / \mathrm{mL}$ of protocatechuic acid (PCA) or $0.008 \mathrm{mg} / \mathrm{m}$ of quercetin (Q) could extend the lifespan of the wild-type strain by 5 days at $50 \%$ viability compared to untreated strain (Fig. 1c). During chronological aging, the viability of cells pretreated with antioxidants Q or PCA or the extract showed positive effects, possibly due to the anti-oxidation potential of antioxidants. They may trigger the anti-aging system of yeast cells to gain better resistance to toxic or accumulated free radicals.

The Sir2 signaling pathway and the anti-aging effect of antioxidants and extract. Sir2 regulates NAD-dependent deacetylase, which participates in a wide range of cellular events, including chromosome silencing, chromosome segregation, DNA recombination and the determination of life-span ${ }^{32}$. It is also involved in the transcriptional repression of the silent mating-type loci, HML and HMR, and telomeric silencing via the association with Sir3 and Sir $4^{33}$. First, the ROS detection experiments and spot assays were performed to identify the oxidation states of cells and to test whether the long-lived $\Delta$ sir2 strain is oxidative stress resistant. The wild-type and the $\Delta$ sir2 strains were either pre-treated or untreated with antioxidants Q and PCA or Hom Dang rice bran extract (HD) and then exposed to $5 \mathrm{mM}$ hydrogen peroxide $\left(\mathrm{H}_{2} \mathrm{O}_{2}\right)$ for $1 \mathrm{hr}$. The untreated $\Delta$ sir 2 strain showed higher ROS accumulation when compared to the untreated wild-type strain by approximately $45 \%$ (Fig. 2a). Pre-treatment with antioxidants Q or PCA or the extract (HD) by approximately $22 \%, 0 \%$, or 56\%, respectively, 

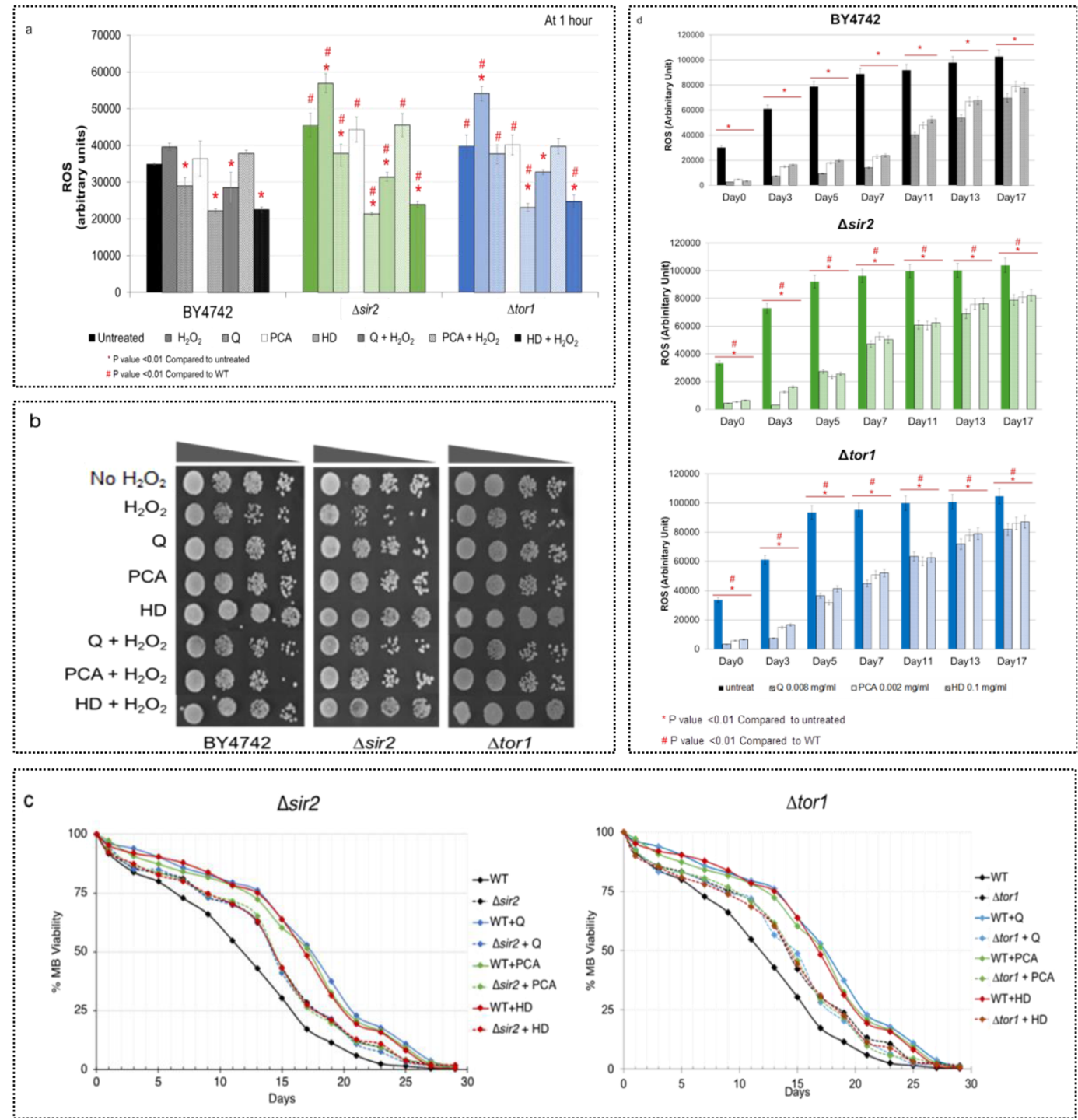

Figure 2. (a) Survival curves of chronologically aging yeast cells from day 0 to day 30 were shown. Lack of Tor 1 or Sir2 significantly increased the longevity of S. cerevisiae. Pre-treatment with antioxidants quercetin (Q) or protocatechuic acid (PCA) or Hom Dang rice bran extract (HD) showed increasing life-span extension only in the WT strain but not in the deletion mutants of Tor 1 or Sir2. $\left({ }^{*} p,{ }^{*} p<0.01\right.$, two-tailed Student's t test compared to untreated condition and wild-type, respectively). Data were presented as means $\pm \operatorname{SEM}(n=9)$. (b) Quantification of cellular ROS levels via the fluorescent DCFH-DA assay in the S. cerevisiae WT and yeast mutants $\Delta$ sir 2 or $\Delta$ tor 1 . Cells were treated with antioxidants $0.008 \mathrm{mg} / \mathrm{mL}$ of quercetin $(\mathrm{Q}), 0.002 \mathrm{mg} / \mathrm{mL}$ of protocatechuic acid (PCA) or $0.1 \mathrm{mg} / \mathrm{mL}$ Hom Dang rice bran extract (HD). After, cells were exposed to $5 \mathrm{mM}$ of hydrogen peroxide $\left(\mathrm{H}_{2} \mathrm{O}_{2}\right)$ for $1 \mathrm{hr}$. to induce generation of ROS. Endogenous ROS levels of cells were measured. Error bars represented standard error of the mean (SEM) (* $p,{ }^{\#} p<0.01$, two-tailed Student's $\mathrm{t}$ test compared to untreated condition and wild-type, respectively). (c) The oxidative stress resistance of the WT, the $\Delta$ sir 2 and the $\Delta$ tor 1 strains were examined via spot assays. Cells were first treated or untreated with antioxidants quercetin (Q), protocatechuic acid (PCA) or Hom Dang rice bran extract (HD), and then exposed to $5 \mathrm{mM}$ hydrogen peroxide $\left(\mathrm{H}_{2} \mathrm{O}_{2}\right)$ for $1 \mathrm{hr}$. prior to be spotted on YPD plates. Growth was examined after incubation in a dark room at $30^{\circ} \mathrm{C}$ for $48 \mathrm{hr}$. Three independent experiments were done. (d) Quantification of intracellular ROS levels in the chronological ageing S. cerevisiae WT, the $\Delta$ sir2 and the $\Delta$ tor 1 strains treated with antioxidants $0.008 \mathrm{mg} / \mathrm{mL}$ of quercetin $(\mathrm{Q}), 0.002 \mathrm{mg} / \mathrm{mL}$ of protocatechuic acid (PCA), or $0.1 \mathrm{mg} / \mathrm{mL}$ Hom Dang rice bran extract (HD). The ROS levels were analyzed by the fluorescent DCFH-DA assay. Error bars represented standard error of the mean (SEM) $\left({ }^{*} p,{ }^{*} p<0.01\right.$, two-tailed Student's t test compared to untreated condition and wild-type, respectively).

decreased the ROS accumulation in the $\Delta$ sir2 strain and optimized growth (Fig. 2a,b). Following the exposure to $\mathrm{H}_{2} \mathrm{O}_{2}$, the highest ROS level and increased sensitivity was found for the $\Delta$ sir2 strain by approximately $21 \%$ (Fig. 2a,b). The antioxidants Q, PCA and especially the Hom Dang rice bran extract (HD), could lower the ROS 


\begin{tabular}{|c|c|c|c|c|c|c|c|c|c|c|c|c|c|c|c|c|c|}
\hline \multirow[b]{2}{*}{ Strain } & \multirow[b]{2}{*}{ Day } & \multicolumn{4}{|c|}{$\begin{array}{l}\text { \% Normalized ROS levels compared to } \\
\text { day } 0\end{array}$} & \multicolumn{4}{|c|}{$\begin{array}{l}\text { \% Difference in ROS levels normalized } \\
\text { to day } 0\end{array}$} & \multicolumn{4}{|c|}{$\begin{array}{l}\text { \% Difference in ROS levels normalized to } \\
\text { untreated condition }\end{array}$} & \multicolumn{4}{|c|}{$\begin{array}{l}\text { \% Difference in ROS levels normalized } \\
\text { to WT }\end{array}$} \\
\hline & & untreated & \begin{tabular}{|l|} 
Quercetin \\
(Q)
\end{tabular} & PCA & $\begin{array}{l}\text { HD } \\
\text { extract }\end{array}$ & untreated & \begin{tabular}{|l|}
$\begin{array}{l}\text { Quercetin } \\
\text { (Q) }\end{array}$ \\
\end{tabular} & PCA & \begin{tabular}{|l} 
HD \\
extract
\end{tabular} & untreated & \begin{tabular}{|l|} 
Quercetin \\
(Q)
\end{tabular} & PCA & $\begin{array}{l}\text { HD } \\
\text { extract }\end{array}$ & untreated & $\begin{array}{l}\text { Quercetin } \\
\text { (Q) }\end{array}$ & PCA & $\begin{array}{l}\text { HD } \\
\text { extract }\end{array}$ \\
\hline \multirow{7}{*}{ WT } & 0 & 100 & 100 & 100 & 100 & 0 & 0 & 0 & 0 & 0 & -91 & -84 & -89 & 0 & 0 & 0 & 0 \\
\hline & 3 & 202 & 279 & 319 & 487 & 50 & 64 & 69 & 79 & 0 & -88 & -75 & -73 & 0 & 0 & 0 & 0 \\
\hline & 5 & 261 & 356 & \begin{tabular}{|l|}
383 \\
\end{tabular} & \begin{tabular}{|l|l|}
587 \\
\end{tabular} & 62 & 72 & 74 & 83 & 0 & -88 & -77 & -75 & 0 & 0 & 0 & 0 \\
\hline & 7 & 294 & 536 & 489 & 705 & 66 & 81 & 80 & 86 & 0 & -84 & -74 & -73 & 0 & 0 & 0 & 0 \\
\hline & 11 & 304 & 1537 & 1023 & 1545 & 67 & 93 & 90 & 94 & 0 & -56 & -48 & -43 & 0 & 0 & 0 & 0 \\
\hline & 13 & 324 & 2044 & 1429 & 1998 & 69 & 95 & 93 & 95 & 0 & -45 & -32 & -31 & 0 & 0 & 0 & 0 \\
\hline & 17 & 340 & 2653 & \begin{tabular}{|l|}
1685 \\
\end{tabular} & 2293 & 71 & 96 & 94 & 96 & 0 & -32 & -23 & -24 & 0 & 0 & 0 & 0 \\
\hline \multirow{7}{*}{$\Delta s i r 2$} & 0 & 100 & 100 & 100 & 100 & 0 & 0 & 0 & 0 & 0 & -87 & -84 & -81 & 10 & 63 & 13 & 85 \\
\hline & 3 & 219 & 68 & 237 & 256 & 54 & -47 & 58 & 61 & 0 & -96 & -83 & -78 & 19 & -60 & -16 & -3 \\
\hline & 5 & 277 & 635 & 441 & 407 & 64 & 84 & 77 & 75 & 0 & -70 & -75 & -72 & 17 & 192 & 30 & 28 \\
\hline & 7 & 290 & 1097 & 995 & 802 & 65 & 91 & 90 & 88 & 0 & -51 & -45 & -48 & 8 & 234 & 130 & 110 \\
\hline & 11 & 300 & 1418 & 1143 & 996 & 67 & 93 & 91 & 90 & 0 & -39 & -39 & -37 & 9 & 51 & 26 & 19 \\
\hline & 13 & 301 & 1609 & 1435 & 1216 & 67 & 94 & 93 & 92 & 0 & -31 & -24 & -24 & 2 & 29 & 13 & 13 \\
\hline & 17 & 313 & 1837 & \begin{tabular}{|l|l|}
1529 \\
\end{tabular} & 1312 & 68 & 95 & 93 & 92 & 0 & -24 & -22 & -21 & 1 & 13 & 3 & 6 \\
\hline \multirow{7}{*}{$\Delta t o r l$} & 0 & \begin{tabular}{|l|l|}
100 \\
\end{tabular} & \begin{tabular}{|l|l|}
100 \\
\end{tabular} & \begin{tabular}{|l|l|}
100 \\
\end{tabular} & \begin{tabular}{|l|l|}
100 \\
\end{tabular} & 0 & 0 & 0 & 0 & 0 & -90 & -83 & -81 & 12 & 22 & 23 & 94 \\
\hline & 3 & 181 & 228 & 260 & 252 & 45 & 56 & 61 & 60 & 0 & -88 & -75 & -73 & 0 & 0 & 0 & 0 \\
\hline & \begin{tabular}{|l|}
5 \\
\end{tabular} & 277 & 1141 & 554 & 629 & 64 & 91 & 82 & 84 & 0 & -61 & -66 & -56 & \begin{tabular}{|l|}
19 \\
\end{tabular} & 292 & \begin{tabular}{|l|}
78 \\
\end{tabular} & 108 \\
\hline & 7 & 282 & 1403 & \begin{tabular}{|l|}
883 \\
\end{tabular} & 790 & 65 & 93 & \begin{tabular}{|l|}
89 \\
\end{tabular} & 87 & 0 & -53 & -47 & -45 & 7 & 220 & 122 & 117 \\
\hline & 11 & 296 & \begin{tabular}{|l|l|}
1974 \\
\end{tabular} & \begin{tabular}{|l|}
1040 \\
\end{tabular} & 951 & 66 & 95 & 90 & 89 & 0 & -37 & -40 & -37 & 9 & 57 & 25 & 19 \\
\hline & 13 & 299 & 2239 & \begin{tabular}{|l|l}
1350 \\
\end{tabular} & 1201 & 67 & 96 & 93 & 92 & 0 & -29 & -23 & -22 & 3 & 34 & 16 & 16 \\
\hline & 17 & 311 & 2551 & 1489 & 1325 & 68 & 96 & 93 & 92 & 0 & -22 & -18 & -17 & 2 & 18 & 9 & 12 \\
\hline
\end{tabular}

Table 4. Normalized ROS levels and difference in ROS levels normalized to day 0 , the untreated condition or WT of CLS assay at day $0,7,13$, and 17 for the $\Delta$ sir 2 and $\Delta$ tor 1 strains. Data used in comparison were significant at $p<0.001$. Q, PCA and HD referred to quercetin, protocathechuic acid and Hom Dang, respectively. Negative values indicated the decreased values.

levels of the $\Delta$ sir 2 strain by approximately $34 \%, 4 \%$, or $54 \%$, respectively, and showed improved growth on the spot tests (Fig. 2a,b). Thus, the $\Delta$ sir2 strain had high intracellular ROS accumulation and showed increased sensitivity to $\mathrm{H}_{2} \mathrm{O}_{2}$ while the antioxidants pre-treatment reduced ROS accumulation and enhanced the growth of the $\Delta$ sir2 strain during the oxidative stress (Fig. 2a,b).

Next, the oxidation states of $\Delta$ sir2 mutant pre-treated or untreated with the antioxidants Q or PCA or the Hom Dang rice bran extract (HD) were investigated to examine the possible correlation between antioxidants and longevity. Deletion of SIR2 resulted in increased percent cell survival and extension of life-span by 2 days at $50 \%$ viability when compared to the untreated wild-type strain (Fig. 2c; dash black line versus solid black line) or approximately $65 \%$ at day 17 . In contrast to the wild-type S. cerevisiae strain with extended life-span of approximately 179-205\%; no life-span extension was found in the antioxidant pre-treated $\Delta$ sir2 strain (Fig. 2a; dash red lines versus dash black line at day 17). To examine the oxidation state of the aging $\Delta$ sir 2 strain in the pre-treatment/un -treatment with antioxidants or the extract, the ROS levels were again measured as cells chronologically aged. As shown, the ROS levels were increased as cells aged (measured from day 0 to day 17) for both the wild-type and $\Delta$ sir 2 strains by approximately $71 \%$ and $68 \%$, respectively (Fig. $2 \mathrm{~d}$ ). Between days $3-11$, in untreated condition, the ROS levels of the $\Delta$ sir2 strain were higher than those of the wild-type strain, suggesting elevated intracellular oxidative stress in cells lacking Sir2 (Fig. 2d). Absence of Sir2 function has been reported to result in a complete loss of transcriptional silencing, an increase in the rate of rDNA repeat and recombination, and a decrease in chromosome stability, leading to defects in the meiotic pachytene checkpoint, which may explain the observed increasing ${ }^{1,4,34,35}$.

Quercetin (Q), protocatechuic acid (PCA), and the extract of Hom Dang rice bran (HD) greatly reduced ROS levels in the wild-type and $\Delta$ sir2 strains, especially during day 3-7 to approximately $45-96 \%$, respectively, in WT (Fig. 2d and Table 4). However, ROS levels remained quite high between days 11 and 17 as cells aged, despite their antioxidant property (Fig. 2d). Despite elevated ROS levels in the $\Delta$ sir 2 strain, its longevity was extended (Fig. 2c,d). Pre-treatment with the antioxidants Q or PCA reduced ROS levels in both the wild-type and $\Delta$ sir2 strains, but only enhanced the longevity of the wild-type strain but not $\Delta \operatorname{sir} 2$ strain (Fig. 2c,d). No clear correlation between the ROS levels, antioxidants and longevity could be found for the $\Delta$ sir 2 strain as no life-span extension was observed upon antioxidant pre-treatment despite a reduction in the ROS level (Fig. 2c,d). Thus, results suggested that the antioxidants could partly reduce ROS levels of the $\Delta$ sir 2 strain.

We reported the identification of a Sir2-independent pathway responsible for the longevity extension associated with antioxidants. Besides, Sir2 and additional mechanisms such as antioxidative defense, which remains intact in the $\Delta$ sir2 strain may operate to reduce the cellular ROS accumulation, may act in parallel pathways to promote longevity in yeast and, perhaps, higher eukaryotes. The higher ROS level observed in the $\Delta$ sir2 strain, as a resulting of cellular activity, may not be harmful to cells but instead protective. Previous work has shown that severe calorie restriction or additional mutations are required to extend the life span of the $\Delta$ sir 2 mutant $^{34}$. Increasing ROS level may be responsible for enhanced longevity in the SIR2 deletion strain through activation 
of the antioxidative system although additional of excessive oxidative stress from $\left(\mathrm{H}_{2} \mathrm{O}_{2}\right)$ exposure may be dangerous. Nevertheless, the Hom Dang rice bran extract showed promising anti-aging property and could replace chemically synthesized pure antioxidants used for ROS reduction and longevity enhancement at a lower cost.

Anti-aging effect and involvement of Tor1 pathway. The Tor1 kinase controls several cellular processes to regulate cell cycle progression from G1 to $S$ phase according to environmental signals ${ }^{36}$. Again, the oxidative stress resistance and spot assays were repeated by exposing the antioxidant-treated and untreated $\Delta$ tor 1 strain to $5 \mathrm{mM}$ hydrogen peroxide for $1 \mathrm{hr}$. The untreated $\Delta$ tor 1 strain showed the highest ROS level as observed for the $\Delta$ sir2 strain (Fig. 2a). This increased ROS level correlated to the observed increased sensitivity (Fig. 2b). The antioxidants and Hom Dang rice bran extract (HD) could reduce the ROS levels of the $\Delta$ tor 1 strain by approximately $37 \%$, resulting in better growth compared to the untreated $\Delta$ tor 1 strain (Fig. $2 \mathrm{a}, \mathrm{b})$. Similarly, these antioxidants could only partly reduce ROS levels as observed. The examination of cell survival, using a spot test, revealed that regardless of increasing ROS level, deletion of TOR1 could overcome the requirement for antioxidant pre-treatment to extend longevity (data not shown). It appeared that increasing ROS level may activate other pathways important for the oxidative defense system. In fact, the cellular function regulated by Tor 1 has been shown to contain a general mechanism involving the sequestration of the oxidative stress response transcription factors Msn2/Msn $4^{37}$. Examination of these factors may provide better understanding of the connection between Tor1-signalling pathways, aging, and oxidative stress response.

Similar treatments were performed using $S$. cerevisiae strain with a deletion of TOR 1 gene. The $\Delta$ tor 1 strain exhibited increased percent survival and could extend the life-span by 2 days at $50 \%$ viability when compared to the wild-type cells under untreated condition (Fig. 2c; dash black versus solid black line). While the antioxidants protocatechuic acid (PCA) and quercetin (Q), or the Hom Dang rice bran extract (HD) further extended the longevity of the wild-type strain, they could not show the same effect in the $\Delta$ tor 1 strain (Fig. 2c; dash red versus solid red line and solid blue and green line versus solid black line). The $\Delta$ tor 1 strain pre-treated with the Hom Dang rice bran extract (HD) did not show the life-span extension when compared with the untreated strain (Fig. 2c; dash red versus solid red line). In addition, the $\Delta$ torl strain pre-treated with PCA, Q or the HD extract could no longer extend its longevity further (Fig. 2c; dash blue and green line versus dash black line). To examine the oxidation state of the aging $\Delta$ tor 1 strain, the ROS levels of cells were again measured for a period of 17 days. Under untreated condition, the $\Delta$ tor 1 strain showed similar ROS level as that of the wild-type strain except for a slightly higher ROS level at day 5 (Fig. 2d). Quercetin (Q), protocatechuic acid (PCA), and Hom Dang rice bran extract (HD) could reduce the ROS levels of $\Delta$ tor 1 strain by approximately $17-22 \%$ at day 17 , although the ROS level remained higher than that of the wild-type strain under this condition (Fig. $2 \mathrm{~d}$ ). This indicated that the longevity effects of the Hom Dang rice bran extract and antioxidants resembles to Tor1 deletion in life-span extension.

Roles of longevity factors in oxidative stress resistance. Again, the ROS levels of strains lacking longevity kinases Sch9 or Rim15, and transcription factors Msn2, Msn4 or Asg1 were examined following treatment with antioxidants and then with hydrogen peroxide $\left(\mathrm{H}_{2} \mathrm{O}_{2}\right)$ for a duration of $10 \mathrm{~min}$ or $1 \mathrm{hr}$. As shown, these mutants showed rapid elevated ROS levels after a 10-min exposure to $\mathrm{H}_{2} \mathrm{O}_{2}$ (Fig. 3a; upper panel). Treatments with quercetin (Q) or protocatechuic acid (PCA) did not reduce the ROS levels (Fig. 3a; upper panel). Examination of cell survival via spot assays showed that the short exposure to $\mathrm{H}_{2} \mathrm{O}_{2}(10 \mathrm{~min})$ was not able to inhibit the growth of these deletion mutants of oxidative stress response and in turn induced ROS levels which may immune the cells for better tolerance to oxidative stress (Fig. 3b; upper panel). Also, cells remained viable as there could be a functional redundancy in activating the stress response due to a single gene deletion. Surprisingly, for the longer $\mathrm{H}_{2} \mathrm{O}_{2}$ exposure of $1 \mathrm{hr}$., the ROS levels were reduced greatly, and treatments with antioxidants further decreased the ROS levels (Fig. 3a; lower panel). The observed lower ROS levels were likely due to the inactivation of enzymes for the conversion of the ROS signal to a fluorescence signal as a result of cell death. Since, inactivation of Sch9 may cause up-regulation of many stress-resistance and DNA-repairing genes as well as genes required to extend the chronological lifespan. In fact, the ROS level should be very high under this condition. The long $\mathrm{H}_{2} \mathrm{O}_{2}$ exposure was found to be detrimental for all tested strains, and addition of antioxidants quercetin (Q) or protocatechuic acid (PCA) or the Hom Dang rice bran extract (HD) slightly promoted growth for some strains, such as the $\Delta a s g 1, \Delta$ rim 15 , and $\Delta s c h 9$ strains (Fig. $3 \mathrm{~b}$; lower panel). The correlation between the oxidation state of these mutants and their roles in mediating oxidative stress response was clear. Antioxidant pre-treatment resulted in the lowering ROS levels in these deletion mutants the $\Delta \operatorname{rim} 15$, the $\Delta m s n 2$, the $\Delta m s n 4$ and the $\Delta$ asg1, suggesting that the oxidative stress defense system is a major route for maintaining the cellular ROS level (Fig. 4). The correlation between the oxidation states and longevity of these mutants remains to be further investigated. Likely, their ROS levels would be high due to their direct roles in controlling the oxidative stress response (Fig. 3a) and cellular homeostasis, as observed for shortened longevity of these mutants (Fig. 3c).

Downstream kinases and transcription factors of longevity in S. cerevisiae. Notably, similar effect was observed for the downstream kinases of Tor1 such as Sch9 but not Rim15. Deletion of SCH9 resulted in increased percent cell survival; however, deletion of RIM15 decreased percent cell survival by 2 and 3 days at $50 \%$ viability, respectively when compared to the untreated wild-type strain (Fig. 3c). Unlike the wild-type strain (Fig. 3c), antioxidants quercetin (Q) and protocatechuic acid (PCA) or Hom Dang rice bran extract (HD) could not extend the longevity of the $\Delta s c h 9$ strain, but could extend that of the $\Delta$ rim 15 strain (Fig. $3 \mathrm{c}$ ). Msn2 and Msn 4 are positive transcriptional factors that act as a component of the stress responsive system. They recognize and bind to the stress response element (STRE) in response to various forms of stress (heat, oxidative, osmotic shock and others $)^{35,38,39}$. Deletion of MSN2 or MSN4 genes exhibited decreased percent cell survival by 3 days at $50 \%$ viability when compared to the untreated wild-type strain (Fig. 3a; dash black versus solid black line). The $\Delta m s n 2$ 


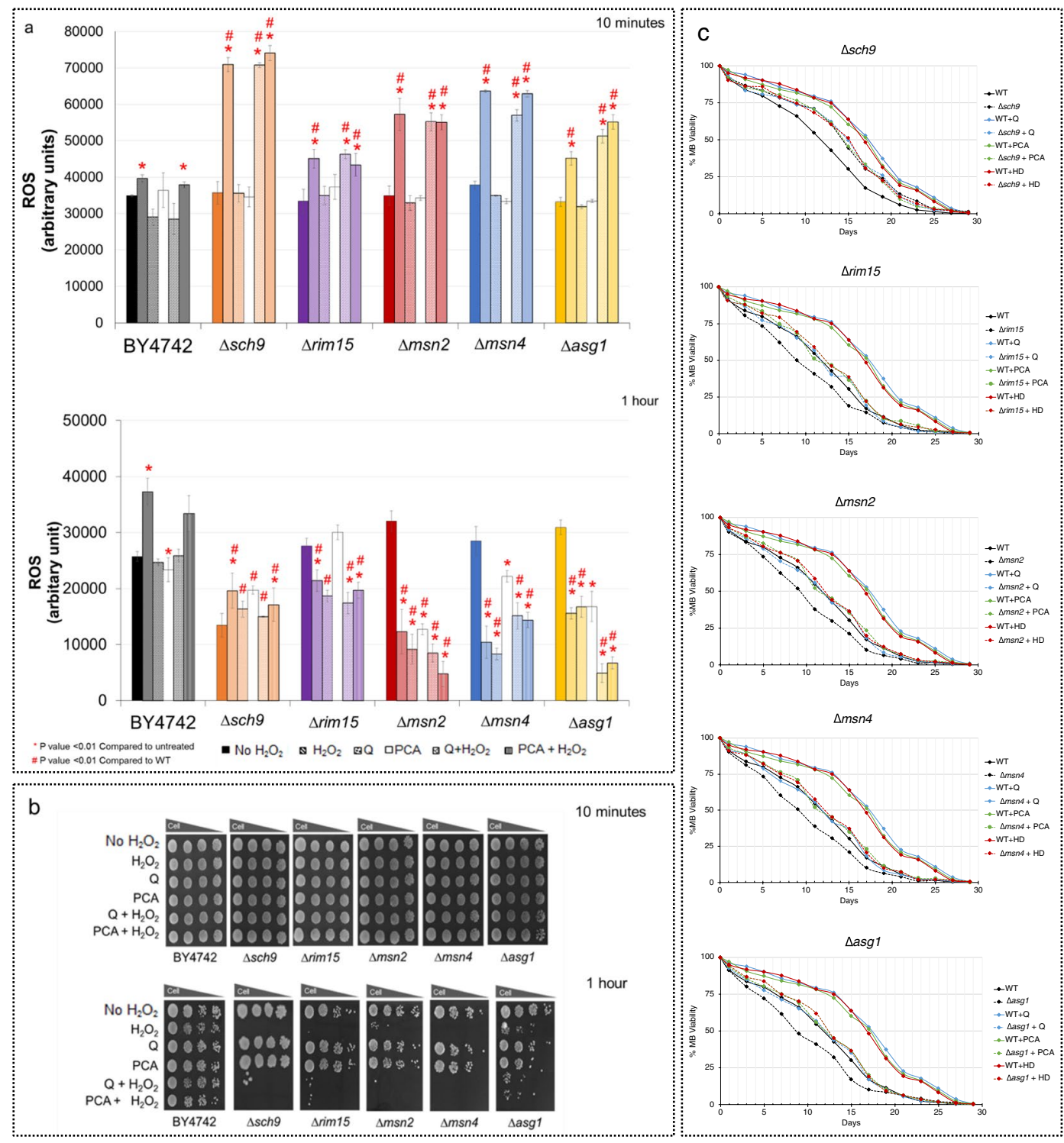

Figure 3. (a) Quantification of cellular ROS levels via the fluorescent DCFH-DA assay in the S. cerevisiae WT and yeast mutants $\Delta s c h 9$, the $\Delta \operatorname{rim} 15$, the $\Delta m s n 2$, the $\Delta m s n 4$ and the $\Delta a s g 1$ strains. Strains were treated with antioxidants $0.008 \mathrm{mg} / \mathrm{mL}$ of quercetin (Q), $0.002 \mathrm{mg} / \mathrm{mL}$ of protocatechuic acid (PCA) or $0.1 \mathrm{mg} / \mathrm{mL}$ Hom Dang rice bran extract (HD). After, cells were exposed to $5 \mathrm{mM}$ of hydrogen peroxide $\left(\mathrm{H}_{2} \mathrm{O}_{2}\right)$ for 10 mins (upper panel) or $1 \mathrm{hr}$ (lower panel) to induce generation of ROS. Endogenous ROS levels of cells were measured. Error bars represent standard error of the mean (SEM) $\left({ }^{*} p,{ }^{*} p<0.01\right.$, two-tailed Student's t test compared to untreated condition and wild-type, respectively). (b) The oxidative stress resistance of the WT, the $\Delta$ sir2 and the $\Delta$ tor 1 strains were examined via spot assays. Cells were first treated or untreated with antioxidants quercetin (Q), protocatechuic acid (PCA) or Hom Dang rice bran extract (HD), and then exposed to $5 \mathrm{mM}$ hydrogen peroxide $\left(\mathrm{H}_{2} \mathrm{O}_{2}\right)$ for 10 mins (upper panel) or $1 \mathrm{hr}$ (lower panel) prior to be spotted on YPD plates. Growth was examined after incubation in a dark room at $30^{\circ} \mathrm{C}$ for $48 \mathrm{hr}$. Three independent experiments were done. (c) The life-spans of yeast deletion mutants lacking longevity factors Sch9, Rim15, Msn2, Msn4 or Asg1 in the presence and absence of antioxidants quercetin (Q), protocatechuic acid (PCA) or Hom Dang rice bran extract (HD). Survival curves of chronologically aging yeast cells from day 0 to day 30 were shown. Lack of Sch9 significantly increased the longevity while, in contrast, lack of Rim15, Msn2, Msn4 or Asg1 significantly decreased the longevity of S. cerevisiae. Pretreatment with antioxidants quercetin (Q) or protocatechuic acid (PCA) or Hom Dang rice bran extract (HD) showed increasing life-span extension in the WT, the $\Delta \operatorname{rim} 15$, the $\Delta m s n 2$, the $\Delta m s n 4$ and the $\Delta a s g 1$ strains. $\left({ }^{*} p,{ }^{\#} p<0.01\right.$, two-tailed Student's t test compared to untreated condition and wild-type, respectively). Data were presented as means $\pm \operatorname{SEM}(\mathrm{n}=9)$.

or $\Delta m s n 4$ strain pre-treatment with quercetin (Q), protocatechuic acid (PCA), or the Hom Dang rice bran extract (HD) showed an increase in life-span extension by approximately $78-121 \%, 78-127 \%$, and $179-205 \%$, respectively, as compared to the wild-type treated strain at day 17 (Fig. 3c; dash blue, green and red lines versus solid 


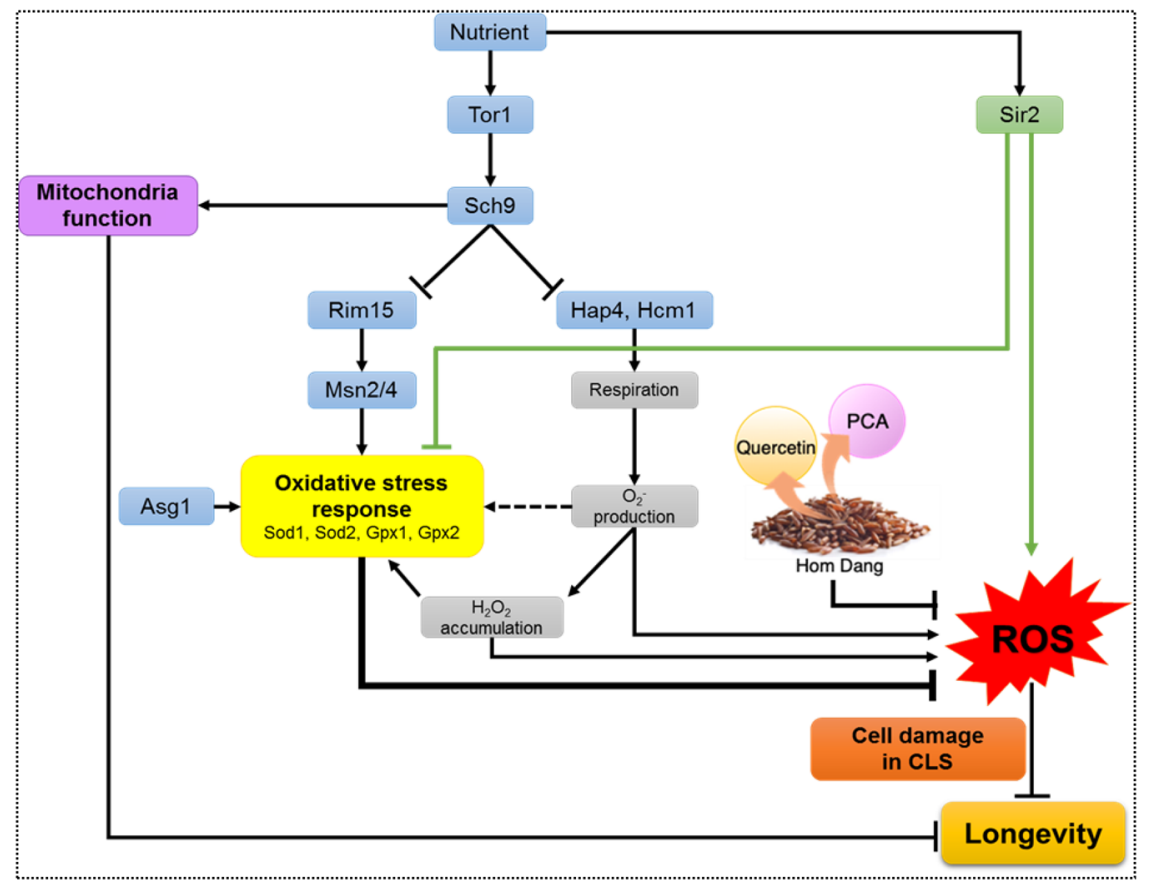

Figure 4. Proposed model to describe modes of actions of antioxidants quercetin (Q), protocatechuic acid (PCA) or Hom Dang rice bran extract (HD) to reduce intracellular ROS accumulation and roles of longevity factors of the Tor1- and Sir2-signaling pathways and transcription factors of the oxidative stress response system to reduce or promote longevity in the model yeast S.cerevisiae.

black line). Lastly, Asg1 is a recently characterized zinc cluster transcription factor of stress response ${ }^{40}$. Null mutant asg1 has a respiratory deficiency, calcofluor white sensitivity and slightly increased cycloheximide resistance $^{41}$. Deletion of $A S G 1$ also exhibited reduced percentage cell survival by 3 days at $50 \%$ viability when compared to the untreated wild-type strain (Fig. 3a; dash black versus solid black line). Likewise, the $\Delta a s g 1$ strain supplemented with tested antioxidants or Hom Dang rice bran extract (HD) could increase the life-span by approximately $78-121 \%$ at day 17 (Fig. 3a; dash blue, green and red lines versus solid black line).

\section{Discussion}

Rice bran is produced as an agricultural byproduct of white rice processing. This nutrient-rich outer hull of rice kernel offers a number of health benefits as an excellent source of essential nutrients including antioxidants, vitamins, minerals, and fiber. The concentration of bioactive compounds and antioxidants present in rice bran or other cereal grains is significantly dependent on the grain color ${ }^{22}$. Among cereal grains, rice bran has more phenolic content and antioxidant activity than wheat, sorghum, corn and barley that could be applied in food and health industries ${ }^{42}$. The TPC of red and black rice bran extracts correlated well with the antioxidant activities obtained via the DPPH, ABTS radical scavenging ability, and Ferric ion reducing power (Table 1). The reported antioxidant activity could be attributed to the different kinds of antioxidants in the pigmented rice bran, as has been reported by other studies ${ }^{35,38}$, and could promote human health by reducing the concentrations of reactive cell-damaging free radicals. They act as antioxidants as singlet oxygen quencher and radical scavenger ${ }^{39}$.

The protective effects of protocatechuic acid and quercetin against oxidative stress via a direct antioxidant mechanism through free radical-scavenging activity and their anti-inflammatory activity have been documented $^{43}$. For example, phenolic acids including protocatechuic acid, found only in red and black rice bran, and $p$-coumaric acid, found only in red rice bran, function as antioxidants and convert the superoxide anion to $\mathrm{H}_{2} \mathrm{O}_{2}$ by hydrogen donation and oxidization of hydroxyl radical to water ${ }^{14}$. This study shows that extracts of red and black rice bran restore the viability of yeast cells lacking the antioxidant enzyme, superoxide dismutases and glutathione peroxidases (Fig. 1a). The Hom Dang rice bran extract, quercetin and protocatechuic acid, also decrease ROS levels in the yeast cells up to day 21 after pre-treatment, implicating the anti-aging intervention (Fig. 1b). These results are consistent with previous studies, which confirm that proanthocyanidin found in Hom Dang rice bran (Table 2) increases the antioxidant capacity by stimulating the enzymatic activities of superoxide dismutase and catalase ${ }^{44}$.

Also, Hom Dang rice bran contains compounds with antioxidant and anti-inflammatory activities in accordance with the inhibition of protonation of the superoxide anion and the hydroxyl radical initiation of lipid peroxidation, which support the finding that these antioxidants could help to restore damaged cell membranes (Fig. 1d). The previous report also showed that Hom Dang rice bran extract can scavenge the superoxide anion and hydroxyl radicals due to its bioactive compounds contents ${ }^{45}$. These results well corroborate a previous finding that quercetin significantly suppresses the level of malondialdehyde, a naturally end product of lipid peroxidation and an oxidative stress biomarker, which could contribute to the protective effect on cell membranes ${ }^{42}$. In 
addition, quercetin found in the Hom Dang rice bran (Table 2) has been shown to act as estrogenic agonist which activates estrogen activity to inhibit cell proliferation in human breast cancer ${ }^{46}$. Therefore, rice bran extract has a wide spectrum of health benefits and pharmacological functions $s^{27,47,48}$. Some medical studies have also revealed that high consumption of whole grain could reduce the risk of coronary disease ${ }^{35}$. Protocatechuic acid could be spontaneously degraded by microbial colon and function as anti-colorectal cancer ${ }^{49}$. Some studies have claimed that phenolic compounds from rice bran found in the intestine act to promote enzymatic digestion and absorption of nutrients, thereby preventing colorectal cancer and chronic diseases ${ }^{50}$.

Due to mammalian analogy, S. cerevisiae is widely used as a model for aging studies to elucidate new targets and cellular mechanisms ${ }^{51}$. A number of antioxidants and natural compounds, including resveratrol, quercetin, and tanshinone, has been shown to extend the life-span of S. cerevisiae ${ }^{52-54}$. The increase in cell viability under stress conditions has been linked to longevity ${ }^{14}$. Here, antioxidants found in pigmented rice extracts (Table 2) and some signaling and stress responsive factors are shown to display longevity effect in the yeast model of ageing (Fig. 3). These important factors determine life-span extension in a very complex fashion. The extract of Hom Dang rice bran or antioxidants quercetin or protocatechuic acid delays chronological aging and extends the life-span significantly via a reduction of the ROS accumulation (Figs. 2 and 3). The stress responsive kinases and transcription factors play an important role in altering ROS accumulation (Figs. 2 and 3). With respect to longevity promotion, in addition to its anti-oxidative property, antioxidants may influence longevity through modulation of the expression of target genes related to SIR2 of the sirtuin family or the TOR pathway, as is the case in mammals ${ }^{55,56}$. They may directly or indirectly function by activating the Rim 15 -mediated stress response system to enhance the production of antioxidant enzymes for reduction of ROS level (Fig. 4). Further investigation will be required. Nevertheless, the results suggest that Hom Dang rice bran extract and the antioxidants mimic the inactivation effect of Sir2, Tor 1 or Sch9 for reduction of ROS level and compensate for the activation of stress response system as shown for Rim15, Msn2, Msn4 or Asg1 deletion (Fig. 4).

The antioxidant pre-treatment promotes longevity in the absence of Rim15 kinase. Tor1 kinase activates Sch9 and functions to inhibit Rim15. In addition, inactivation of Sch9, a homolog of the mammalian threonine protien-kinase Akt, increases stress resistance and extends the chronological life span of non-diving cells through activation of respiration via Hap4 and $\mathrm{Hcm}^{37,57}$.

This leads to the production of superoxide radicals that are further converted to hydrogen peroxide whose accumulation then signals to activate the stress response system for the reduction of ROS and cell damages thereby increasing life-span extension (Fig. 4). Sch9 also plays another important role in mediating mitochondrial functions which affects oxidative stress and is related to the chronological life-span (Fig. 4). The accumulated ROS may activate downstream components of Rim15, including transcription Msn2/4 and Asg1, for activation of antioxidant enzymes associated with longevity ${ }^{19}$ (Fig. 4). Importantly, this study identifies a new role of the transcription factor Asg1. It not only functions in stress response but also acts as a longevity factor as observed for the Msn2/4 transcription factors of the TOR signaling pathway (Fig. 4). Together, they activate the expression of antioxidant enzymes to decrease ROS accumulation and restore cellular damages during exposure to oxidative stress (Fig. 4). In addition, Asg1 is required for full activation of genes in several pathways of fatty acid synthesis, including $\beta$-oxidation (POX1, FOX2, and POT1) and triacylglycerol breakdown (TGL3), which affect cellular lipid contents and plasma membrane permeability ${ }^{44}$. As shown, plasma membrane permeability could be preserved in strains pre-treated with the extract or antioxidants (Fig. 1d). Further the roles of these new longevity factors in life-span extension, particularly in relation to lipid metabolism, need to be explored.

In addition to tested compounds, it has been reported that protocatechuic acid and proanthocyanidin are able to elevate catalase and superoxide dismutase activities, and treatment with rutin present in red rice bran (Table 2) could elevate the transcription levels of genes encoding MnSod and catalase, while reducing the transcription level of Tor $^{22}$. Furthermore, catechin present in the red rice bran (Table 2) also acts as a powerful hydrogen-donating radical scavenger of ROS and RNS and chelates divalent transition metal ions $\left(\mathrm{Cu}^{2+}, \mathrm{Zn}^{2+}\right.$ and $\mathrm{Fe}^{2+}$ ), thereby preventing the $\mathrm{Fe}^{2+}$-induced formation of free radicals ${ }^{58}$. It also increases the expression of manganese superoxide dismutase SOD2 gene in Drosophila melanogaster and inhibits the signaling of transcription factor NF- $\kappa \mathrm{B}$, leading to reductions in liver and kidney damage, and improvement of age-associated inflammation and oxidative stress in mice ${ }^{45}$. Thus, the evidence provided here and in other studies clearly support the antioxidative-assisted anti-aging effects of the pigmented rice extract and antioxidant compounds.

The discovery of new natural antioxidants with longevity effect could offer advantageous strategies for the protection against harmful radicals, chronic inflammation, and age-related diseases ${ }^{59,60}$. It is, therefore, necessary to explore ways to utilize agricultural by-products, such as pigmented rice bran, as food, dietary supplements, or healthcare products to promote our health and overall well-being ${ }^{27,47,48}$. Further investigation in yeast may serve as a useful model to elucidate conserved mechanisms of aging and age-related diseases in humans.

\section{Materials and Methods}

Materials. The two Thai pigmented rice cultivars, namely Hom Dang (KMDL105R-PSL-E-14) and Kum Doi Saket (KKU2012-PS-PANPB-OS-001), were kindly supplied by S\&J International Enterprises Public Co., Ltd., Bangkok, Thailand. The following chemicals were used for the preparation of yeast growth medium (YNB) $1 \%$ yeast extract (Aldrich-Sigma, USA), 2\% peptone (Aldrich-Sigma, USA), 2\% glucose (Aldrich-Sigma, USA), and 2\%agar (Aldrich-Sigma, USA). S. cerevisiae mutant strains: BY4742 (WT) and single deletion strains obtained from Open Biosystem (Dharmacon, Inc., Lafayette, CO, USA).

Microbial and treatments. The yeast cells were grown in the synthetic minimal YNB medium initially containing $2 \%$ glucose, in the presence of quercetin $0.008 \mathrm{mg} / \mathrm{mL}$ (Aldrich-Sigma, USA), protocatechuic 
acid $0.002 \mathrm{mg} / \mathrm{mL}$ (Aldrich-Sigma, USA), and Hom Dang rice bran extracts concentration at $(1,0.1,0.01$ and $0.001 \mathrm{mg} / \mathrm{mL}$ ) (S\&J International Enterprises Public Company Limited).

Determination bioactive compounds by UPLC MS/MS. An ultraperformance liquid chromatography (UPLC), Waters Acquity (Milford, MA, USA), system was coupled with a triplequadrupole-linear ion trap tandem mass spectrometer (Applied Biosystems 4000 Q TRAP; Life Technologies Corporation, Carlsbad, CA, USA) with an electrospray ionisation (ESI) source. $\mathrm{A} \mathrm{C}_{18}$ reversed phase Acquity column $(1.7 \mu \mathrm{m}, 150 \mathrm{~mm} \times 4.6 \mathrm{~mm})$. The mobile phase was a binary solvent system consisting of solvent A (water with $0.1 \%$ formic acid) and solvent $\mathrm{B}\left(\mathrm{CH}_{3} \mathrm{CN}\right)$. The UPLC gradient for mass screening was $0-5 \mathrm{~min}, 90 \% \mathrm{~A} ; 5-15 \mathrm{~min}, 90-10 \% \mathrm{~A} ; 15-20 \mathrm{~min}$, $10 \% \mathrm{~A} ; 20-25 \mathrm{~min}, 10-90 \% \mathrm{~A} ; 25-30 \mathrm{~min}, 90 \% \mathrm{~A}$. The flow rate was $0.25 \mathrm{~mL} / \mathrm{min}$, and the injection volume was $10 \mu \mathrm{L}$. All samples were filtered with $0.2 \mu \mathrm{m}$ nylon membrane filter prior to injection. The mass spectra were acquired from $\mathrm{m} / \mathrm{z} 100-1000$ with a $20 \mathrm{~ms}$ ion accumulation time. All mass spectrometric data were acquired in positive ionisation mode. The capillary and voltage of the ESI source were maintained at $400^{\circ} \mathrm{C}$ and $-4.5 \mathrm{kV}$, respectively. The scan rate was $1000 \mathrm{amu} / \mathrm{s}$. Data acquisition and data processing was performed using Analyst 1.4.2. MS Fragmenter 12.0 (Advanced Chemistry Development, Toronto, Canada) was used to predict compound fragmentation $^{61}$.

Determination of total phenolic content (TPC). The total phenolic content of each fraction was determined using the Folin-Ciocalteu method with some modifications. Briefly, $50 \mu$ l of extract solution was shaken for $1 \mathrm{~min}$ with $1 \mathrm{~mL}$ of diluted (1:10 with water) Folin-Ciocalteu reagent. After the mixture was shaken, $50 \mu \mathrm{L}$ of $10 \%$ Sodium carbonate $\left(\mathrm{Na}_{2} \mathrm{CO}_{3}\right)$ was added, and the final volume was made up to $5 \mathrm{~mL}$ with distilled water. After 2 hour of reaction, the absorbance at $760 \mathrm{~nm}$ was determined and used to estimate the phenolic acid content using a standard curve prepared using gallic acid ${ }^{61}$.

Free-radical scavenging activity on DPPH. Total free radical scavenging capacity of the extracts from two pigmented rice bran extracts: red and black was estimated according to the previously reported method with slight modification using the stable DPPH radical, which has an absorption maximum at wavelength of $517 \mathrm{~nm}$. All the determinations were performed in triplicate. The capability to scavenge the DPPH radical was calculated using the following equation. DPPH Scavenged $(\%)=\left(\mathrm{A}_{517}\right.$ of sample- $\mathrm{A}_{517}$ of control/ $\mathrm{A}_{517}$ of control $) \times 100(1)$, where, $A_{517}$ of control is absorbance at $t=0 \mathrm{~min} ; A_{517}$ of sample is absorbance at $t=30$ min. ${ }^{61}$.

2,2'-azino-bis-3-ethylbenzthiazoline-6-sulphonic acid cation decoloration (ABTS) assay. 2,2'-azino-bis-3-ethylbenzthiazoline-6-sulphonic acid (ABTS) assays was prepared by mixing ANTS $(7 \mathrm{mM})$ with potassium persulfate $(2.45 \mathrm{mM})$ in volume ratio of $1: 1$. The solution was stored in dark condition 6 hours, and dilute with ethanol to the absorbance of $0.7 \pm 0.02$ at $734 \mathrm{~nm}$. The experiment was carried out by $0.2 \mathrm{~mL}$ extract to $1.8 \mathrm{~mL}$ of diluted ABTS + solution. The absorbance was recorded after 20 minutes incubation. The affinity of sample to quench free radical was evaluated. The results were expressed as value of $\mathrm{IC}_{50}$. All samples were tested in triplicate. The calculation is stated as scavenging $\%=[(\mathrm{A}$ of sample- $\mathrm{A}$ of control)/A of control] $\times 100 \%{ }^{61}$.

Ferric reducing antioxidant power activity (FRAP). The chelating capacity of extracts measured using ferrous ion $\left(\mathrm{Fe}^{3+}\right)$ reduction assay. Sample $(0.1 \mathrm{~mL})$ with the concentration range from $20-100 \mu \mathrm{g} / \mathrm{mL}$ was dissolved in $2.5 \mathrm{~mL}$ of sodium buffer $(0.2 \mathrm{M}, \mathrm{pH} 6.6)$ before adding $1 \% \mathrm{w} / \mathrm{v}$ potassium ferricyanide III $(2.5 \mathrm{~mL})$. After the mixture was incubated at $50^{\circ} \mathrm{C}$ for 20 minutes, $2.5 \mathrm{~mL}$ trichloroacetic $(10 \% \mathrm{w} / \mathrm{v})$ was added into the mixture. The upper layer of solution $(2.5 \mathrm{~mL})$ was diluted with distilled water $(2.5 \mathrm{~mL})$ and then added with iron(III)chloride $(0.1 \% \mathrm{w} / \mathrm{v}) 500 \mu \mathrm{L}$ then measure at $700 \mathrm{~nm}^{31,61}$.

Spot assay. WT and mutant strains (the $\Delta \operatorname{sod} 1, \Delta \operatorname{sod} 2, \Delta g p x 1, \Delta g p x 2, \Delta \operatorname{ctt} 1, \Delta \operatorname{cta} 1, \Delta$ tor $1, \Delta \operatorname{sir} 2, \Delta$ sch 9 , $\Delta$ rim15, $\Delta m s n 2, \Delta m s n 4$ or $\Delta$ asg1 strains) were first grown overnight. After, cells were regrown to $\mathrm{OD}_{600}$ of 0.6 ., $0.001,0.01$, or $0.1 \mathrm{mg} / \mathrm{mL}$ pigmented rice bran extract or $(0.008 \mathrm{mg} / \mathrm{mL})$ quercetin $(\mathrm{Q}),(0.002 \mathrm{mg} / \mathrm{mL})$ protocatechuic acid (PCA) were then added to the mixture of $100 \mathrm{ul}$ of cells, followed by the exposure to $5 \mathrm{mM} \mathrm{H}_{2} \mathrm{O}_{2}$ for 10 mins. or $1 \mathrm{hr}$. at $30^{\circ} \mathrm{C}$ before being spotted on YPD agar medium and incubated in a dark room at $30^{\circ} \mathrm{C}$ for $48 \mathrm{hr}$.

Dichloro-dihydro-fluorescein diacetate (DCFH-DA) assay for ROS detection. Endogenous ROS levels of $S$. cerevisiae cells were measured by a fluorometric assay using $2^{\prime}, 7^{\prime}$-dichlorofluorescein diacetate (DCFH-DA) (Sigma-Aldrich) as a ROS indicator, as previously described ${ }^{62}$. For chronological study, WT, $\Delta$ tor 1 and $\Delta$ sir2 strains were inoculated overnight then diluted to $\mathrm{OD}_{600}$ of 0.1 . Antioxidants or the rice bran extract was added to the cell cultures at the final concentration of $(0.008 \mathrm{mg} / \mathrm{mL})$ quercetin $(\mathrm{Q}),(0.002 \mathrm{mg} / \mathrm{mL})$ protocatechuic acid (PCA) or $0.1 \mathrm{mg} / \mathrm{mL}$ pigmented rice bran extract. Cells were incubated at $30^{\circ} \mathrm{C}$ from day 0 at the $\mathrm{OD}_{600}$ of 0.6 to day 17. For the oxidative stress assay, WT, $\Delta \operatorname{tor} 1, \Delta \operatorname{sir} 2, \Delta \operatorname{sch} 9, \Delta \operatorname{rim} 15, \Delta m s n 2, \Delta m s n 4$ or $\Delta$ asg1 strains were first pretreated with the antioxidants or the rice extract prior to be exposed to 10 mins. or $1 \mathrm{hr}$. of $5 \mathrm{mM} \mathrm{H}_{2} \mathrm{O}_{2}$ at $30^{\circ} \mathrm{C}$. Then, $5 \mathrm{~mL}$ of cell were treated with $(30 \mu \mathrm{L}$ of $10 \mathrm{mM})$ DCFH-DA and incubated at $37^{\circ} \mathrm{C}$ for 30 minutes. The cells were harvested as described $\mathrm{in}^{62}$. ROS induction was measured using a fluorescent microplate reader (excitation $490 \mathrm{~nm}$, emission $535 \mathrm{~nm}$ ). The protein content of the samples was measured using Bradford assay, and the protein concentration used to normalize the ROS induction of each sample.

Plasma Membrane Permeability assay. Propiodium Iodine (PI, Thermo Fisher Scientific, Taiwan) staining assay performed on yeast cells. Yeast strains were at to log phase in YPD. After cells were treated with 
$(0.008 \mathrm{mg} / \mathrm{mL})$ of quercetin, $(0.002 \mathrm{mg} / \mathrm{mL})$ of protocatechuic acid, $(0.1$ or $0.01 \mathrm{mg} / \mathrm{mL})$ Hom Dang rice bran extract (HD) grown to day 1 or day 7 . The supernatant was removed and, the cells were washed three times with PBS with $0.01 \%$ Tween 20 and then staining with PI solution (Sigma-Aldrich) at $5 \mu \mathrm{g} / \mathrm{mL}$ of final concentration and then incubated in the dark place at $37^{\circ} \mathrm{C}$ for 30 minutes. The cells were washed 3 times and observed under the fluorescence microscope (IX83, Inverted Microscopes, Olympus, Japan) ${ }^{62}$.

Chronological lifespan assay. Wild-type and mutants cells were grown in the synthetic minimal YNB medium initially containing $2 \%$ glucose in the presence or absence of extract or antioxidants quercetin or protocatechuic acid at the final concentrations of $(0.1 \mathrm{mg} / \mathrm{mL}),(0.008 \mathrm{mg} / \mathrm{mL})$ and $(0.002 \mathrm{mg} / \mathrm{mL})$, respectively. The cellular viability was determined by the methylene blue technique using Thomas's counting chamber ${ }^{51}$. The number of stained (non-active cells) or un-stained (active cells) and the number of buds were counted in five different fields with a total of at least 200-300 cells. The percentage of 'viable' cells (\%MB 'viability') was the number of un-stained cells (live cells) divided by the total number of cells (stained and unstained). Under the conditions used, mean viability was estimated with an accuracy of $10 \%{ }^{63}$.

Statistical analysis. Data are presented as means $\pm \operatorname{SEM}(n=9 ; * p<0.01$; the $\mathrm{p}$ values for comparing the means of two groups were calculated using the IBM SPSS statistics software using t-test). Also, data are presented as means $\pm \operatorname{SEM}\left(\mathrm{n}=9 ;{ }^{*} p<0.01 \text {; the } p \text { values for comparing }\right)^{63}$.

Received: 14 June 2019; Accepted: 13 October 2019;

Published online: 02 December 2019

\section{References}

1. Botstein, D., Chervitz, S. A. \& Cherry, J. M. Yeast as a model organism. Science. 277, 1259-1260 (1997).

2. Longo, V. D. \& Fabrizio, P. Chronological aging in Saccharomyces cerevisiae. Subcell Biochem. 57, 101-121 (2012).

3. Canakci, C. F., Cicek, Y. \& Canakci, V. Reactive oxygen species and human inflammatory periodontal diseases. Biochemistry (Mosc). 70,619-628 (2005).

4. Valko, M. et al. Free radicals and antioxidants in normal physiological functions and human disease. Int J Biochem Cell Biol. 39, 44-84 (2007).

5. Devasagayam, T. P. et al. Free radicals and antioxidants in human health: current status and future prospects. $J$ Assoc Physicians India. 52, 794-804 (2004)

6. Diezmann, S. Oxidative stress response and adaptation to $\mathrm{H} 2 \mathrm{O} 2$ in the model eukaryote Saccharomyces cerevisiae and its human pathogenic relatives Candida albicans and Candida glabrata. Fungal Biol. Rev. 28, 126-136 (2014).

7. Persson, T., Popescu, B. O. \& Cedazo-Minguez, A. Oxidative stress in Alzheimer's disease: why did antioxidant therapy fail? Oxid Med Cell Longev. 2014, 427318 (2014).

8. Bokoch, G. M. \& Diebold, B. A. Current molecular models for NADPH oxidase regulation by Rac GTPase. Blood. 100, 2692-2696 (2002).

9. Shahidi, F. \& Wanasundara, P. K. Phenolic antioxidants. Crit Rev Food Sci Nutr. 32, 67-103 (1992).

10. Goffman, F. \& Bergman, C. Rice Kernel phenolic content and its relationship with antiradical efficiency. J Sci Food Agr. 84 (2004).

11. Shibuya, N. Phenolic acids and their carbohydrate esters in rice endosperm cell walls. Phytochemistry. 23, 2233-2237 (1984).

12. Munzel, T., Gori, T., Bruno, R. M. \& Taddei, S. Is oxidative stress a therapeutic target in cardiovascular disease? Eur Heart J. 31, 2741-2748 (2010).

13. Cicero, A. F. \& Gaddi, A. Rice bran oil and gamma-oryzanol in the treatment of hyperlipoproteinaemias and other conditions. Phytother Res. 15, 277-289 (2001).

14. Goufo, P. \& Trindade, H. Rice antioxidants: phenolic acids, flavonoids, anthocyanins, proanthocyanidins, tocopherols, tocotrienols, gamma-oryzanol, and phytic acid. Food Sci Nutr. 2, 75-104 (2014).

15. Zhang, M. W., Zhang, R. F., Zhang, F. X. \& Liu, R. H. Phenolic profiles and antioxidant activity of black rice bran of different commercially available varieties. J Agric Food Chem. 58, 7580-7587 (2010).

16. Lin, J.-Y. \& Tang, C.-Y. Determination of total phenolic and flavonoid contents in selected fruits and vegetables, as well as their stimulatory effects on mouse splenocyte proliferation. Food Chemistry. 101, 140-147 (2007).

17. Ryu, S. N., Park, S. Z. \& Ho, C. T. High performance liquid chromatographic determination of anthocyanin pigments in some varieties of black rice. J Food Drug Anal. 6, 729-736 (1998).

18. Irakli, M. N., Samanidou, V. F., Biliaderis, C. G. \& Papadoyannis, I. N. Development and validation of an HPLC-method for determination of free and bound phenolic acids in cereals after solid-phase extraction. Food Chem. 134, 1624-1632 (2012).

19. Qiu, Y., Liu, Q. \& Beta, T. Antioxidant properties of commercial wild rice and analysis of soluble and insoluble phenolic acids. Food Chem. 121, 140-147 (2010).

20. Chandrasekara, A. \& Shahidi, F. Content of insoluble bound phenolics in millets and their contribution to antioxidant capacity. $J$ Agric Food Chem. 58, 6706-6714 (2010).

21. Srinivasan, M., Sudheer, A. R. \& Menon, V. P. Ferulic Acid: therapeutic potential through its antioxidant property. J Clin Biochem Nutr. 40, 92-100 (2007).

22. Apel, K. \& Hirt, H. J. A. R. P. B. Reactive oxygen species: metabolism, oxidative stress, and signal transduction. Annu Rev Plant Biol. 55, 373-399 (2004).

23. Herrero, E., Ros, J., Bellí, G. \& Cabiscol, E. Redox control and oxidative stress in yeast cells. Biochim Biophys Acta. 1780, $1217-1235$ (2008).

24. Tanaka, T., Izawa, S. \& Inoue, Y. GPX2, encoding a phospholipid hydroperoxide glutathione peroxidase homologue, codes for an atypical 2-Cys peroxiredoxin in Saccharomyces cerevisiae. J Biol Chem. 280, 42078-42087 (2005).

25. Ookhtens, M. \& Kaplowitz, N. Role of the liver in interorgan homeostasis of glutathione and cyst(e)ine. Semin Liver Dis. 18, 313-329 (1998).

26. Sampaio-Marques, B., Burhans, W. C. \& Ludovico, P. Yeast at the Forefront of Research on Ageing and Age-Related Diseases. Prog Mol Subcell Biol. 58, 217-242 (2019).

27. Prasad, M. N. N., Sanjay, K. \& Khatokar, M. S. Health Benefits of Rice Bran - A Review. Journal of Nutrition \& Food Sciences. 01 (2011).

28. Barzegar, A. \& Moosavi-Movahedi, A. A. Intracellular ROS protection efficiency and free radical-scavenging activity of curcumin. PLoS One. 6, e26012 (2011).

29. Ayala, A., Parrado, J., Bougria, M. \& Machado, A. Effect of oxidative stress, produced by cumene hydroperoxide, on the various steps of protein synthesis. Modifications of elongation factor-2. J Biol Chem. 271, 23105-23110 (1996).

30. Pavelescu, L. A. On reactive oxygen species measurement in living systems. J Med Life. 8(Spec Issue), 38-42 (2015) 
31. Cui, H., Kong, Y. \& Zhang, H. Oxidative stress, mitochondrial dysfunction, and aging. J Signal Transduct. 2012, 646354 (2012).

32. Wierman, M. B. \& Smith, J. S. Yeast sirtuins and the regulation of aging. FEMS Yeast Res. 14, 73-88 (2014).

33. Rusche, L. N., Kirchmaier, A. L. \& Rine, J. The establishment, inheritance, and function of silenced chromatin in Saccharomyces cerevisiae. Annu Rev Biochem. 72, 481-516 (2003).

34. Fabrizio, P. et al. Sir2 blocks extreme life-span extension. Cell. 123, 655-667 (2005).

35. Aune, D. et al. Whole grain consumption and risk of cardiovascular disease, cancer, and all cause and cause specific mortality: systematic review and dose-response meta-analysis of prospective studies. Bmj. 353, i2716 (2016).

36. Perez-Hidalgo, L. \& Moreno, S. Coupling TOR to the Cell Cycle by the Greatwall-Endosulfine-PP2A-B55 Pathway. Biomolecules. 7 (2017).

37. Qie, B. et al. Sch9 regulates intracellular protein ubiquitination by controlling stress responses. Redox Biol. 5, 290-300 (2015).

38. Muntana, N., Sriseadka, T. \& Mahatheeranont, S. Quantification of carotenoid and flavonoid components in brans of some Thai black rice cultivars using supercritical fluid extraction and high-performance liquid chromatography-mass spectrometry. Journal of Food Lipids. 15, 488-503 (2008).

39. Tournaire, C. et al. Antioxidant activity of flavonoids: efficiency of singlet oxygen (1 delta g) quenching. J Photochem Photobiol B. 19, 205-215 (1993).

40. Siripat Jansuriyakul, Pichayada Somboon, Napachai Rodboon, Olena Kurylenko, Andriy Sibirny, Nitnipa Soontorngun, (2016) The zinc cluster transcriptional regulator Asg1 transcriptionally coordinates oleate utilization and lipid accumulation in Saccharomyces cerevisiae. Applied Microbiology and Biotechnology 100 (10):4549-4560 (2016).

41. Akache B, Wu K, Turcotte B. Phenotypic analysis of genes encoding yeast zinc cluster proteins. Nucleic Acids Res. 29(10), 2181-90 (2001).

42. Sun, T. \& Ho, C.-T. In Functional Food and Health Vol. 993 ACS Symposium Series Ch. 13, 143-150 (American Chemical Society, 2008).

43. Bao, D., Wang, J., Pang, X. \& Liu, H. Protective Effect of Quercetin against Oxidative Stress-Induced Cytotoxicity in Rat Pheochromocytoma (PC-12) Cells. Molecules. 22 (2017).

44. Masella, R., Di Benedetto, R., Vari, R., Filesi, C. \& Giovannini, C. Novel mechanisms of natural antioxidant compounds in biological systems: involvement of glutathione and glutathione-related enzymes. J Nutr Biochem. 16, 577-586 (2005).

45. Sinthorn, W., Chatuphonprasert, W., Chulasiri, M. \& Jarukamjorn, K. Thai red rice extract provides liver protection in paracetamoltreated mice by restoring the glutathione system. Pharm Biol. 54, 770-779 (2016).

46. Batra, P. \& Sharma, A. K. Anti-cancer potential of flavonoids: recent trends and future perspectives. 3 Biotech. 3, 439-459 (2013).

47. Somnuk, S., Komindr, S., Munkong, N., Parklak, W. \& Lerdvuthisopon, N. Protective effects of rice bran water extract (RBE) on hepatic fat metabolism and oxidative damage in rats fed a high-fat diet. J Med Assoc Thai. 100, 224-233 (2017).

48. Somintara, S., Leardkamolkarn, V., Suttiarporn, P. \& Mahatheeranont, S. Anti-Tumor and Immune Enhancing Activities of Rice Bran Gramisterol on Acute Myelogenous Leukemia. PLoS One. 11, e0146869 (2016).

49. Quinn, L. et al. Sinapinic and protocatechuic acids found in rapeseed: isolation, characterisation and potential benefits for human health as functional food ingredients. Irish J Agr Food Res. 56, 104 (2017).

50. Brown, D. G., Borresen, E. C., Brown, R. J. \& Ryan, E. P. Heat-stabilised rice bran consumption by colorectal cancer survivors modulates stool metabolite profiles and metabolic networks: a randomised controlled trial. BrJ Nutr. 117, 1244-1256 (2017).

51. Kwolek-Mirek, M. \& Zadrag-Tecza, R. Comparison of methods used for assessing the viability and vitality of yeast cells. FEMS Yeast Research. 14, 1068-1079 (2014).

52. Howitz, K. T. et al. Small molecule activators of sirtuins extend Saccharomyces cerevisiae lifespan. Nature. 425, 191-196 (2003).

53. Belinha, I. et al. Quercetin increases oxidative stress resistance and longevity in Saccharomyces cerevisiae. J Agric Food Chem. 55, 2446-2451 (2007).

54. Wu, Z., Song, L., Liu, S. Q. \& Huang, D. Tanshinones extend chronological lifespan in budding yeast Saccharomyces cerevisiae. Appl Microbiol Biotechnol. 98, 8617-8628 (2014).

55. Vassallo, N. Polyphenols and health: new and recent advances. (Nova Publishers, 2008).

56. Dilberger, B. et al. Polyphenols and Metabolites Enhance Survival in Rodents and Nematodes-Impact of Mitochondria. Nutrients. 11, $1886(2019)$

57. Weinberger, M. et al. Growth signaling promotes chronological aging in budding yeast by inducing superoxide anions that inhibit quiescence. Aging (Albany NY). 2, 709-726 (2010).

58. Singh, B. N., Shankar, S. \& Srivastava, R. K. Green tea catechin, epigallocatechin-3-gallate (EGCG): mechanisms, perspectives and clinical applications. Biochem Pharmacol. 82, 1807-1821 (2011).

59. Pan, M. H., Lai, C. S., Tsai, M. L., Wu, J. C. \& Ho, C. T. Molecular mechanisms for anti-aging by natural dietary compounds. Mol Nutr Food Res. 56, 88-115 (2012).

60. Wickens, A. P. Ageing and the free radical theory. Respir Physiol. 128, 379-391 (2001).

61. Chew, C. Y., Chua, L. S., Soontorngun, N. \& Lee, C. T. Discovering potential bioactive compounds from Tualang honey. Agriculture and Natural Resources. 52, 361-365 (2018).

62. Somboon, P. et al. Fungicide Xylaria sp. BCC 1067 extract induces reactive oxygen species and activates multidrug resistance system in Saccharomyces cerevisiae. Future Microbiol. 12, 417-440 (2017).

63. Lutchman, V. et al. Six plant extracts delay yeast chronological aging through different signaling pathways. Oncotarget. 7 , 50845-50863 (2016).

\section{Acknowledgements}

We would like to gratefully acknowledge to National Research Council of Thailand (NRCT), NRCT scholarship under the new researchers development according to the direction of research and innovation 2019 scholarship, Petchra Pra Jom Klao scholarship and King Mongkut's University of Technology Thonburi for financial supports. We also acknowledge S\&J International Enterprises Public Company Limited for provision of pigmented rice extracts.

\section{Author contributions}

Conceived and designed the study and experiments: P.Su. and N.S. Performed the experiments: P.Su., P.So and R.S. Analyzed the data: P.Su., R.P., M.U., C.L.S., P.So. and N.S. Contributed reagents/materials/analysis tools: M.U., C.L.S.and N.S. Wrote the paper: P.Su. and N.S. All authors reviewed the manuscript.

\section{Competing interests}

The authors declare no competing interests. 
Additional information

Correspondence and requests for materials should be addressed to N.S.

Reprints and permissions information is available at www.nature.com/reprints.

Publisher's note Springer Nature remains neutral with regard to jurisdictional claims in published maps and institutional affiliations.

(c) (i) Open Access This article is licensed under a Creative Commons Attribution 4.0 International License, which permits use, sharing, adaptation, distribution and reproduction in any medium or format, as long as you give appropriate credit to the original author(s) and the source, provide a link to the Creative Commons license, and indicate if changes were made. The images or other third party material in this article are included in the article's Creative Commons license, unless indicated otherwise in a credit line to the material. If material is not included in the article's Creative Commons license and your intended use is not permitted by statutory regulation or exceeds the permitted use, you will need to obtain permission directly from the copyright holder. To view a copy of this license, visit http://creativecommons.org/licenses/by/4.0/.

(C) The Author(s) 2019 